\title{
Interaction effects and phase relaxation in disordered systems
}

\author{
I.L. Aleiner ${ }^{1,2}$, B.L. Altshuler ${ }^{3,4}$, and M.E. Gershenson ${ }^{5}$ \\ ${ }^{1}$ Ruhr-Universität-Bochum, Bochum, D-44780, Germany \\ ${ }^{2}$ Department of Physics and Astronomy, SUNY at Stony Brook, Stony Brook, NY 11794 \\ ${ }^{3}$ NEC Research Institute, 4 Independence Way, Princeton, NJ 08540 \\ ${ }^{4}$ Physics Department, Princeton University, Princeton, NJ 08544 \\ ${ }^{5}$ Serin Physics Laboratory, Rutgers University, Piscataway, NJ 08854-8019
}

(October 15, 2018)

\begin{abstract}
This paper is intended to demonstrate that there is no need to revise the existing theory of the transport properties of disordered conductors in the so-called weak localization regime. In particular, we demonstrate explicitly that recent attempts to justify theoretically that the dephasing rate (extracted from the magnetoresistance) remains finite at zero temperature are based on the profoundly incorrect calculation. This demonstration is based on a straightforward evaluation of the effect of the electron-electron interaction on the weak localization correction to the conductivity of disordered metals. Using well-controlled perturbation theory with the inverse conductance $g$ as the small parameter, we show that this effect consists of two contributions. First contribution comes from the processes with energy transfer smaller than the temperature. This contribution is responsible for setting the energy scale for the magnetoresistance. The second contribution originates from the virtual processes with energy transfer larger than the temperature. It is shown that the latter processes have nothing to do with the dephasing, but rather manifest the second order (in $1 / g$ ) correction to the conductance. This correction is calculated for the first time. The paper also contains a brief review of the existing experiments on the dephasing of electrons in disordered conductors and an extended qualitative discussion of the quantum corrections to the conductivity and to the density of electronic states in the weak localization regime.
\end{abstract}

PACS numbers: 73.20.Fz, 73.20.Jc, 73.23.-b,

\section{INTRODUCTION}

At low temperatures, the classical conductivity of disordered conductors (normal metals and semiconductors) is determined by the scattering of electrons off a quenched disorder (e.g., impurities and defects). This residual conductivity is given by the Drude formula:

$$
\sigma=\frac{e^{2} n \tau}{m}=\frac{e^{2} n l}{p_{F}}
$$

where $v_{F}$ and $p_{F}$ are the Fermi velocity and Fermi momentum of the electrons, correspondingly, $m$ and $n$ denote their mass and density, $\tau$ is the transport elastic mean free time, while $l=v_{F} \tau$ is the mean free path of electrons.

This expression can be justified provided the elastic mean free path $l$ is sufficiently large, since all the corrections to the classical Drude conductivity (1.1) are of the order of $\hbar /\left(p_{F} l\right) \ll 1$. However, in low dimensions, these quantum corrections to the conductivity (QCC) diverge when temperature $T$ decreases. Eventually, they drive the system to the insulating regime.

QCC are of a substantial importance even for conductors that are far from the strong localization regime: in a wide range of parameters QCC, though being smaller than the classical conductivity (1.1), determine all the temperature and magnetic field dependences of the conductivity. The systematic study of QCC was started almost two decades ago. The comprehensive review of the status of the problem from both theoretical and experimental viewpoints can be found in several paperst

According to their physical origin QCC, can be divided into two distinct groups. The correction of the first type, known as the weak localization (WL) correction, is caused by the quantum interference effect on the diffusive motion of a single electron. For low dimensional $(d=1,2)$ infinite systems, WL QCC diverges, and this divergence should be regularized either by magnetic field or by some other dephasing mechanism.

The second type of QCC, usually referred to as the interaction effects, are absent in the one-particle approximation; they are entirely due to interactions between electrons. These corrections can be interpreted as the scattering of an electron off the inhomogeneous distribution of the density of the rest of the electrons. One can attribute this inhomogeneous distribution to Friedel oscillations produced by each impurity. The role of the electron-electron interactions in this type of QCC is to produce a static self-consistent (and temperature-dependent) potential. Such a 
potential does not lead to any real transitions between single-electron quantum states. Therefore, it does not break the $T$-invariance of the system and neither affects nor regularizes the WL corrections. We will elaborate more on this point in Sec. II.

However, the interactions between electrons are by no means irrelevant to WL QCC. Indeed, these interactions cause phase relaxation of the single-electron states, and thus result in the cut-off the divergences in WL corrections. This dephasing requires real inelastic collisions between the electrons. When the temperature $T$ and magnetic field $H$ are low enough, this dephasing provides the leading mechanism to regularize QCC of the weak localization type. This means that in this regime interactions between electrons can not be taken into account perturbatively: the higher is the term of the perturbation theory expansion the stronger it diverges. For the reasons, which will become clear below, in this paper we restrict ourselves by the lowest order of the perturbation theory in the interactions. This is possible, provided the dephasing and, consequently, the WL effects are mostly determined by the external magnetic field. Under these conditions interactions between electrons modify the dephasing rate slightly, and thus cause temperature dependence of the magnetoresistance. As a result, we end up with two classes of interaction contributions to the conductivity: corrections to WL QCC due to the interactions (class I) and the genuine interaction QCC (class II).

Recently, there were several attempts to revise this copyentional picture. Based on their own measurements, as well as on experimental results of other groups, authors of 1 t argued that apparent saturation of the dephasing rate at $T \rightarrow 0$ has an intrinsic nature - scattering of the electrons by "zero-point fluctuations of electromanetic environment". This suggestion was claimed to be put onto more "theoretical grounds" by Golubev and Zaikin 1 .

Even though these suggestions (as we strongly believe and aim to explain below) can be rejected using purely physical arguments, they still generate a sympathy in some part of the physical community. The situation becomes worrisome, since the paperl, at the first glance, looks like a respectable calculation an experimentalist could rely on. Since it is much easier to observe the saturation rather than not to observe it (a possible reason for this saturation is discussed, e.g., in Ref. 10), one can expect that in the future, quite a number of experimental and non-experimental papers confirming the role of the zero-point motion in the dephasing mechanism will appear. Having all this in mind, we have undertaken in this paper a straightforward analysis of the effect of electron-electron interactions on the weak localization. We explicitly demonstrated that the dephasing (i.e. the characteristic scale of the magnetoresistance) is determined by the real processes with the energy transfer smaller or of the order of temperature. The role of the processes with the transmitted energy larger than temperature (which was referred to as "zero-point motion" in Refs. 6 9) is to produce type two QCC. The explicit formula for those corrections in the second order of the perturbation theory in the inverse dimensionless conductance has been obtained for the first time.

The paper is organized as follows. Section II reviews qualitatively physics of the quantum corrections to the conductivity. In the same section we infppduce the notion of the dephasing time $\tau_{\varphi}$. Section III briefly outlines the essential content of the "new theories" idea of our calculation and the results, which clearly contradict to all the conclusions of Refs. 8.9. Technical details of this calculation are described in section V. Section V1 is devoted to a critical analysis of Refs. 8, 9. We discuss their results and possible sources of mistakes made in these papers. In the same section we show that the results of these references disagree with the available experimental data in two- and three- dimensional systems by several orders of magnitude and the agreement in one-dimensional case claimed in Ref. 11 is a pure coincidence in the region of parameters were the weak localization theory is already not applicable. Our findings are summarized in Conclusion.

\section{QUANTUM CORRECTIONS TO THE CONDUCTIVITY: A QUALITATIVE PICTURE}

This section contains only rather old results and ideas. We review them here in spite of the fact that there is a number of review articles that discuss in details both qualitative and technical parts of the theory of disordered conductors in the weak localization regime 1 目. The main reason to do this is to make this paper self-contained, so that any reasonably educated physicist can read it without consulting the reviews continuously, and to underline the main physical concepts which are being rejected by Refs. 69. We also propose somewhat new intuition about the physical origin of the effects of the electron-electron interaction. Nevertheless, experts in the field can skip this section.

\section{A. Weak localization}

Anomalous magneforesistance in disordered conductors (doped semiconductors and metals) has been recognized for almost 50 years 12 . For a long time this phenomenon has remained a puzzle. The theoretical understanding of the anomalous magnetoresistance emerged as a spinoff of the theory of Anderson localization. It turned out that the correction to the conductivity, which is due to quantum interference at large length scales, is very sensitive to weak 
magnetic fields. The quantum correction itself may be much smaller than the classical conductivity. Nevertheless, the weak field magnetoresistance is dominated by this correction, and its basic features (its amplitude, dependence on both magnitude and direction of the magnetic field, etc.) are very different from that of the classical magnetoresistance. Since the quantum correction can eventually drive the system to the Anderson insulator, the regime when this correction is small, is called the weak localization (WL) regime, and the theory of the anomalous magnetoresistance is now a part of the theory of weak localization.

A qualitative physical interpretation of WL is usually based on the following arguments, see e.g. Ref. 13. Consider an electron diffusing in a good conductor, $p_{F} l \gg \hbar$. The probability $w$ for the electron to reach, say, point $i$ starting from point $f$, see Fig. 1. can be obtained by, first, finding the semiclassical amplitudes $A_{\alpha}$ for different paths connecting the points, and, second, by squaring the modulus of their sum:

$$
w=\left|\sum_{\alpha} A_{\alpha}\right|^{2}=\sum_{\alpha}\left|A_{\alpha}\right|^{2}+\sum_{\alpha, \beta} A_{\alpha} A_{\beta}^{*} .
$$

The first term in Eq. (2.1) is nothing but the sum of the classical probabilities of the different paths, and the second term is the quantum mechanical interference of the different amplitudes. For generic pairs $\alpha$, $\beta$, the product $A_{\alpha} A_{\beta}^{*}$ oscillates on the scale of the order of $\lambda_{F}=\hbar / p_{F}$ as the function of the position of point $f$. This is because the lengths of paths $\alpha$ and $\beta$ are substantially different. Since all measurable quantities are averaged on the scale much larger than $\lambda_{F}$, such oscillating contributions can be neglected. There are pairs of paths, however, which are coherent. An example of such paths is shown in Fig. 1 $1 \mathrm{~b}$. The paths 1 and 2 coincide almost everywhere but the loop segment $B E B$ (see Fig. $1 \mathrm{~b}$ ) is traversed by trajectories 1 and 2 in the opposite directions. In the absence of the magnetic field and spin-orbit interactions, the phases of the trajectories 1 and 2 are equal. Therefore, the contribution of this paths to the probability $w$ becomes

$$
\left|A_{1}+A_{2}\right|^{2}=\left|A_{1}\right|^{2}+\left|A_{2}\right|^{2}+2 \operatorname{Re} A_{1} A_{2}^{*}=4\left|A_{1}\right|^{2},
$$

i.e. it is twice as large as the classical probability. Thus, in order to determine the value of the weak localization correction to the conductivity $\delta \sigma_{W L}$, one has to determine the classical probability to find such a self-intersecting trajectory.

In order to find this probability, we label all the trajectories by the time $t$ it takes for a classical particle to go around the loop. The classical probability $d P$ that the diffusing particle returns into the phase volume $d V$ at a given time is

$$
d P=\frac{d V}{(D t)^{d / 2}},
$$

where $D=v_{F}^{2} \tau / d$ is the diffusion constant, and $d$ is the number of dimensions. The relevant phase volume can be estimated as $v_{F} d t(\delta \rho \delta \phi)^{d-1}$, where $\delta \rho$ and $\delta \phi$ characterize the transverse distance between the paths 1 and 2 at the intersection point, see Fig. $\mathrm{i} \mathrm{b}$, and $\delta \phi$ is the intersection angle between them. For the interference between paths 1 and 2 to be effective, the uncertainty relation $p_{F} \delta \phi \delta \rho \simeq \hbar$ should hold. We substitute this estimate for $d V$ into Eq. (2.3) and sum up over all the trajectories:

$$
\frac{\delta \sigma}{\sigma} \simeq-\int d P \simeq-v_{F} \lambda_{F}^{d-1} \int_{\tau}^{?} \frac{d t}{(D t)^{d / 2}},
$$

where the negative sign is due to the fact that the returning trajectory should arrive at the intersection point with the momentum almost opposite to the initial one. At low dimensions $d=1,2$, the integral in Eq. (2.4) diverges at the upper limit. Below we will return to the cut-off of this integral.

The explicit calculation first performed in Ref. 14 gives the result similar to Eq. (2.4) up to a numerical factor:

$$
\delta \sigma_{W L}=-\frac{\sigma}{\pi \nu \hbar} \int_{\tau}^{\infty} d t \mathcal{C}(\boldsymbol{r}, \boldsymbol{r} ; t),
$$

where $\sigma$ is defined in Eq. (1.1) and $\nu$ is the density of states per one spin. The retarded classical propagator Cooperon is the solution of the equation

$$
\left(\frac{\partial}{\partial t}-D \nabla_{1}^{2}\right) \mathcal{C}=\delta\left(\boldsymbol{r}_{1}-\boldsymbol{r}_{2}\right) \delta(t)
$$




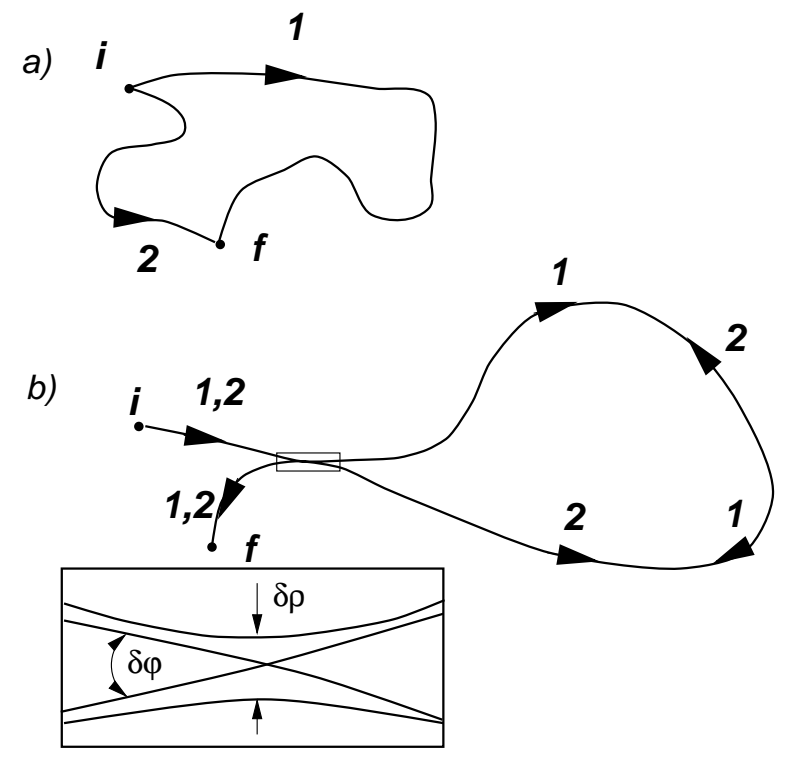

FIG. 1. Examples of the classical (a) non-coherent and (b) coherent paths between points $i$ and $f$. The region of intersection of trajectories is blown up in the inset.

It follows from the previous discussion, that the weak localization correction originates from the quantum interference within the pairs of time reversed paths. Therefore, this correction is extremely sensitive to any violation of the time reversal invariance, e.g. magnetic impurities, external magnetic field, external microwave radiation etc. (we will not consider the spin-orbit interaction in this paper).

Let us discuss first the effect of the magnetic field. The role of the inelastic processes will be considered in the following subsection. The effect of the magnetic field on the conductance is a manifestation of the Aharonov - Bohm effect 15. Let us again consider a pair of the coherent trajectories 1 and 2 from Fig. 1 $1 \mathrm{~b}$. Since those trajectories traverse the loop in the opposite directions, the Aharonov-Bohm phases acquired by them are of the same magnitude, but opposite signs:

$$
A_{1} \rightarrow A_{1} \exp \left(i \frac{e}{c \hbar} \boldsymbol{H} \boldsymbol{S}\right) ; \quad A_{2} \rightarrow A_{2} \exp \left(-i \frac{e}{c \hbar} \boldsymbol{H} \boldsymbol{S}\right)
$$

where $\boldsymbol{S}$ is the directed area of the loop and $\boldsymbol{H}$ is the external magnetic field. As the result, Eq. (2.2) should be modified as $\operatorname{Re} A_{1} A_{2}^{*}=\left|A_{1}\right|^{2} \cos \left(2 \frac{e}{c \hbar} \boldsymbol{H} \boldsymbol{S}\right)$ and, accordingly, Eq. 2.4) now reads

$$
\frac{\delta \sigma}{\sigma} \simeq-v_{F} \lambda_{F}^{d-1} \int_{\tau}^{\infty} \frac{d t}{(D t)^{d / 2}}\left\langle\cos \left(2 \frac{e}{c \hbar} \boldsymbol{H} \boldsymbol{S}\right)\right\rangle \simeq-v_{F} \lambda_{F}^{d-1} \int_{\tau}^{\infty} \frac{d t}{(D t)^{d / 2}} \exp \left(-2 \frac{\left\langle S^{2}(t)\right\rangle}{\lambda_{H}^{4}}\right)
$$

where $\lambda_{H}=\sqrt{\hbar c / e H}$ is the magnetic length. The last transformation in Eq. (2.8) relies on the fact that the area $S_{\alpha}$ swept by the path is the normally distributed random quantity for a diffusive system. For the infinite three-dimensional systems and two-dimensional films in the magnetic field perpendicular to the film plane, $\left\langle S^{2}(t)\right\rangle \simeq(D t)^{2}$. In a $1 \mathrm{~d}$ case (wire) with the transverse dimension $a 17\left\langle S^{2}(t)\right\rangle \simeq(D t) a^{2}$; the same expression holds also for a two-dimensional film in the magnetic field parallel to the film plane with $a$ being the film thickness.

As the result, the weak localization correction is cut-off at the large time limit by $\tau_{H}$ equal to 1 E

$$
\begin{gathered}
\frac{1}{\tau_{H}}=\Omega_{H}=\frac{4 D e H}{\hbar c}, \quad d=3, d=2(\boldsymbol{n} \| \boldsymbol{H}) ; \\
\frac{1}{\tau_{H}}=\frac{D(e H a)^{2}}{3 c^{2} \hbar^{2}}, \quad d=1, d=2(\boldsymbol{n} \perp \boldsymbol{H}),
\end{gathered}
$$

where $\boldsymbol{n}$ is the vector normal to the plane of the film.

More rigorously, the weak localization correction to the conductivity is still given by Eq. (2.5), however, the equation for the Cooperon (2.5) is modified: 


$$
\left[\frac{\partial}{\partial t}+D\left(-i \boldsymbol{\nabla}_{1}-\frac{2 e}{c \hbar} \boldsymbol{A}\left(\boldsymbol{r}_{1}\right)\right)^{2}\right] \mathcal{C}=\delta\left(\boldsymbol{r}_{1}-\boldsymbol{r}_{2}\right) \delta(t),
$$

where the vector potential $\boldsymbol{A}$ due to the magnetic field describes the Aharonov-Bohm phase we have already discussed. For $d=3$ and $d=2(\boldsymbol{n} \| \boldsymbol{H})$, this equation is equivalent to the one for a charged particle in the magnetic field, and $\Omega_{H}$ from Eq. 2.9a) has a meaning of the cyclotron frequency. For one-dimensional systems and films $(\boldsymbol{n} \perp \boldsymbol{H})$, it reduces to

$$
\left[\frac{\partial}{\partial t}-D \frac{\partial^{2}}{\partial x^{2}}+\frac{1}{\tau_{H}}\right] \mathcal{C}=\delta\left(\boldsymbol{r}_{1}-\boldsymbol{r}_{2}\right) \delta(t)
$$

with $\tau_{H}$ being defined by Eq. (2.9b).

Let us now define the dephasing time $\tau_{\varphi}$ and the corresponding length scale $L_{\varphi}=\sqrt{D \tau_{\varphi}}$ in a pure phenomenological way. First, we repeat that the phase itself is not a directly observable quantity, therefore one always has to specify the observable which is sensitive to the phase relaxation. As we have already discussed, the WL correction is cut by the magnetic field. On the other hand, it can also be cut by any process that breaks the phase coherence. We introduce the function $F_{d}(x)$ so that

$$
\delta \sigma_{W L}=\frac{e^{2}}{\hbar} \frac{L_{\varphi}^{2-d}}{d-2} F_{d}\left(\frac{\tau_{\varphi}}{\tau_{H}}\right)
$$

Function $F_{d}(x)$ is different for different dimensionalities $d$, and it has the asymptotic behavior $F_{d}(x)=$ const, $x \ll 1$ and $F_{d}(x) \propto x^{d / 2-1}, x \gg 1$. Therefore, $\tau_{\varphi}$ is defined through the scale of the magnetic fields at which magnetoresistance occurs. Since both the conductivity $\delta \sigma$ and time $\tau_{H}$ can be measured with a great accuracy, one can extract $\tau_{\varphi}$ by fitting the magnetoresistance. In order to define this time with the numerical coefficient, the explicit form of functions $F_{d}(x)$ is needed, which requires some theoretical analysis27. We postpone further discussion of this point until the end of the following subsection.

\section{B. Interaction effects}

It was already mentioned in the introduction that effects of the interaction between the electrons on the conductivity can be subdivided into two groups. The corrections of the first group are associated with scattering of a given electron on the static Friedel - like oscillations of the charge density caused by disorder. These corrections have nothing to do with the energy transfer between the electrons, i.e., with inelastic processes. The second group originates entirely from the inelastic scattering of the electrons. Since the physics involved is completely different for those two groups, we consider them separately.

\section{Correction to the conductivity and tunneling density of states}

First, we will discuss the Hartree type corrections to the tunneling density of states and to the conductivity assuming that the interaction between electrons is determined by a short range two-particle potential $V$. Later we will show how to generalize obtained formulas for the Fock correction and for the Coulomb potential. The rigorous derivation of the results is given in Ref. 1. Here, our goal is to describe the qualitative physical picture.

Consider the local density of states of a non-interacting system

$$
\nu(\epsilon . \boldsymbol{r}) \equiv \sum_{i}\left|\psi_{i}(\boldsymbol{r})\right|^{2} \delta\left(\epsilon-\epsilon_{i}\right),
$$

where $\psi_{i}$ and $\epsilon_{i}$ are the eigenfunctions and eigenenergies of the disordered systems respectively. Hereafter, all the energies are measured from the Fermi energy.

Semiclassically, the density of states 2.13 ) can be presented in the form 18

$$
\nu(\epsilon, \boldsymbol{r}) \approx \text { const }+\operatorname{Im} \sum_{\alpha} A_{\alpha}(\epsilon, \boldsymbol{r}),
$$

where $A_{\alpha}(\epsilon, \boldsymbol{r})$ is the quantum mechanical amplitude corresponding to the path $\alpha$ starting at and returning to the point $\boldsymbol{r}$, see Fig. 2a, and "const" stands instead of a function of energy that is smooth on the scale of the Fermi energy. 
The second term in Eq. (2.14) oscillates on the scale of the order of $\lambda_{F}=\hbar / p_{F}$ as the function of the position of the point $\boldsymbol{r}$, because the lengths of the paths $\alpha, \beta, \gamma$ are much larger than $\lambda_{F}$. As the result, this contribution vanishes upon disorder averaging and there is no interference contribution to the density of states of non-interacting electrons in the disordered metals.

Let us demonstrate now that the interactions between electrons change the situation drastically. In the Hartree approximation, the only effect of the interactions is reduced to the effective one-electron potential $V_{H}$, which should be considered in addition to the random potential of impurities

$$
V_{H}(\boldsymbol{r})=\int d \boldsymbol{r}^{\prime} \rho\left(\boldsymbol{r}^{\prime}\right) V\left(\boldsymbol{r}-\boldsymbol{r}^{\prime}\right) .
$$

Here $\rho(\boldsymbol{r})$ is the electron density and $V\left(\boldsymbol{r}-\boldsymbol{r}^{\prime}\right)$ is the potential of the interaction between two electrons located at the points $\boldsymbol{r}$ and $\boldsymbol{r}^{\prime}$. We assume that this potential is weak and short-range on the scale of the order of the elastic mean free-path $l$. In a clean infinite system, the electron density is homogeneous, and the Hartree correction (2.15) leads only to a uniform (and thus not observable) shift of the chemical potential. In disordered or finite systems, however, the electron density has an inhomogeneous term

$$
\rho(\boldsymbol{r})=\int d \epsilon n_{F}(\epsilon) \operatorname{Im} \sum_{\alpha} A_{\alpha}(\epsilon ; \boldsymbol{r})
$$

which oscillates on the scale of the order of $\lambda_{F}$; for a single impurity these oscillations correspond to the well-known Friedel oscillations. Here, $n_{F}(\epsilon)=1 /\left(1+e^{\epsilon / T}\right)$ is the Fermi-Dirac distribution function.

Scattering of an electron by these density oscillations results in the singularity in the average tunneling density of states. To demonstrate this, consider the scattering off the Hartree potential in the first-order perturbation theory. According to the usual rules of quantum mechanics, the correction to the return amplitude $\delta A$ is given by

$$
\delta A(\epsilon ; \boldsymbol{r}) \approx \int d \boldsymbol{r}^{\prime} V_{H}\left(\boldsymbol{r}^{\prime}\right) \sum_{\beta, \gamma} A_{\beta}\left(\boldsymbol{r}, \boldsymbol{r}^{\prime} ; \epsilon\right) A_{\gamma}\left(\boldsymbol{r}^{\prime}, \boldsymbol{r} ; \epsilon\right)
$$

where $A_{\alpha}\left(\boldsymbol{r}, \boldsymbol{r}^{\prime} ; \epsilon\right)$ is the quantum mechanical amplitude for the electron to get from point $\boldsymbol{r}$ to point $\boldsymbol{r}^{\prime}$ using the classical path $\alpha$, see Fig. [ $b$ b; $A_{\alpha}(\epsilon ; \boldsymbol{r}) \equiv A_{\alpha}(\boldsymbol{r}, \boldsymbol{r} ; \epsilon)$.

a)
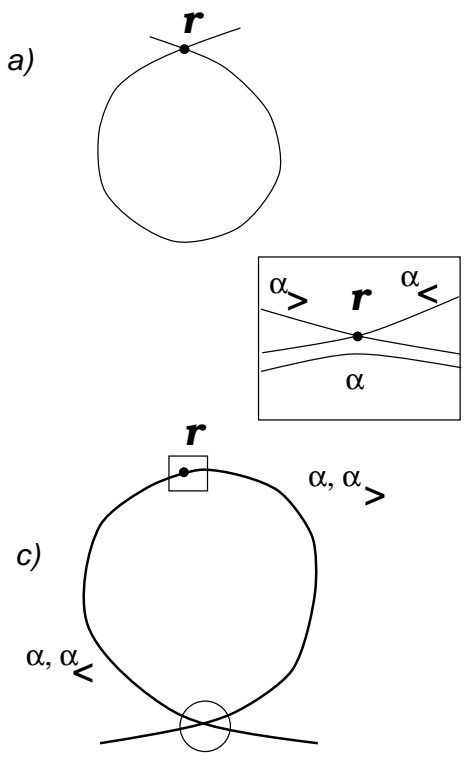
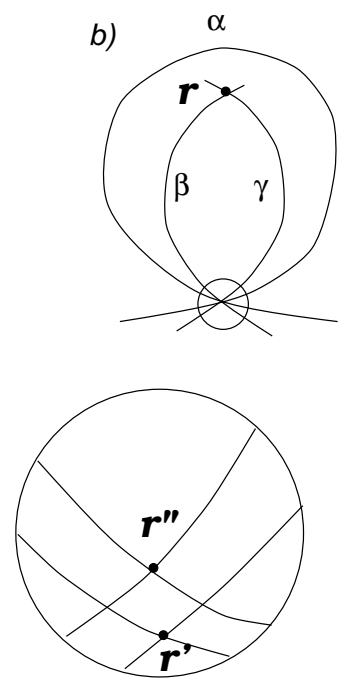

FIG. 2. (a) The returning path that contributes to the density of states $\nu$ and the oscillating part of the electron density $\rho$; (b) Classical paths responsible for the correction to the amplitude of the return; (c) Three paths producing non-vanishing correction to the density of states. The regions of the intersections of the trajectories are blown up in the insets. 
Substituting Eqs. (2.15) and (2.16) into Eq. (2.17), we obtain with the help of Eq. 2.14)

$$
\delta \nu(\epsilon . \boldsymbol{r}) \approx \int d \boldsymbol{r}^{\prime} d \boldsymbol{r}^{\prime \prime} V\left(\boldsymbol{r}^{\prime}-\boldsymbol{r}^{\prime \prime}\right) \int d \epsilon^{\prime} n_{F}\left(\epsilon^{\prime}\right) \sum_{\alpha, \beta, \gamma}\left[\operatorname{Im} A_{\alpha}\left(\epsilon^{\prime}, \boldsymbol{r}^{\prime}\right)\right]\left[\operatorname{Im} A_{\beta}\left(\boldsymbol{r}, \boldsymbol{r}^{\prime \prime} ; \epsilon\right) A_{\gamma}\left(\boldsymbol{r}^{\prime \prime}, \boldsymbol{r} ; \epsilon\right)\right] .
$$

Contribution of a typical set of paths $(\alpha, \beta, \gamma)$ to the sum (2.18) oscillates as the function of $\boldsymbol{r}$. However, similarly to the case of weak localization, there exist special sets of coherent paths, with non-vanishing contributions to the sum. An example of such sets is shown in Fig. 2k. In this case, paths $\gamma$ and $\beta$ are chosen to be parts of the trajectory $\alpha$. We will denote them $\alpha_{<}$and $\alpha_{>}$. Let us neglect in the sum in Eq. (2.18) all the terms except the contributions of the sets $(\alpha, \beta, \gamma)$ of this type: $\left(\beta=\alpha_{<}, \gamma=\alpha_{>}\right.$The phase of the quantum amplitude $A_{\alpha}$ of a path $\alpha$ is connected with the classical action $\mathcal{S}_{\alpha}$ which corresponds to this path: $A_{\alpha} \propto e^{i \mathcal{S}_{\alpha}} / \hbar$. Note that difference between the energies $\epsilon$ and $\epsilon^{\prime}$ is small, and that points $\boldsymbol{r}^{\prime}$ and $\boldsymbol{r}^{\prime \prime}$ are close due to the short range nature of the potential $V$. Expansion of $\mathcal{S}_{\alpha}$ in coordinate and energy with the use of identities

$$
-\frac{\partial \mathcal{S}_{\alpha}\left(\boldsymbol{r}_{1}, \boldsymbol{r}_{2} ; \epsilon\right)}{\partial \epsilon}=t_{\alpha} ; \quad \frac{\partial \mathcal{S}_{\alpha}\left(\boldsymbol{r}_{1}, \boldsymbol{r}_{2} ; \epsilon\right)}{\partial \boldsymbol{r}_{1}}=\boldsymbol{p}_{\alpha}^{f} ; \quad \frac{\partial \mathcal{S}_{\alpha}\left(\boldsymbol{r}_{1}, \boldsymbol{r}_{2} ; \epsilon\right)}{\partial \boldsymbol{r}_{2}}=\boldsymbol{p}_{\alpha}^{i}
$$

( $t_{\alpha}$ is the time it takes for an electron with energy $\epsilon$ to go along the path $\alpha$ from the initial point $\boldsymbol{r}_{2}$ to the final point $\boldsymbol{r}_{1}$, while $\boldsymbol{p}_{(1,2)}^{(\alpha)}$ are respectively its final and initial momenta) allows to rewrite $A_{\alpha}\left(\epsilon^{\prime}, \boldsymbol{r}^{\prime}\right)$ as

$$
A_{\alpha}\left(\epsilon^{\prime}, \boldsymbol{r}^{\prime}\right)=A_{\alpha}\left(\epsilon, \boldsymbol{r}^{\prime \prime}\right) e^{i t_{\alpha}\left(\epsilon-\epsilon^{\prime}\right)} \exp \left[i\left(\boldsymbol{r}^{\prime}-\boldsymbol{r}^{\prime \prime}\right) \cdot\left(\boldsymbol{p}_{2}^{(\alpha)} \boldsymbol{p}_{1}^{(\alpha)}\right)\right]
$$

As a result, Eq. (2.18) acquires the form

$$
\delta \nu(\epsilon . \boldsymbol{r}) \approx \sum_{\alpha} \int d \boldsymbol{r}^{\prime} V\left(\boldsymbol{r}^{\prime}\right) \exp \left[i \boldsymbol{r}^{\prime}\left(\boldsymbol{p}_{\alpha}^{f}-\boldsymbol{p}_{\alpha}^{i}\right)\right] \operatorname{Re} \int d \boldsymbol{r}^{\prime \prime} \int d \epsilon^{\prime} n_{F}\left(\epsilon^{\prime}\right) e^{i t_{\alpha}\left(\epsilon-\epsilon^{\prime}\right)} A_{\alpha}\left(\epsilon, \boldsymbol{r}^{\prime \prime}\right) A_{\alpha_{<}}^{*}\left(\boldsymbol{r}, \boldsymbol{r}^{\prime \prime} ; \epsilon\right) A_{\alpha_{>}}^{*}\left(\boldsymbol{r}^{\prime \prime}, \boldsymbol{r} ; \epsilon\right)
$$

To further simplify equation (2.19), we notice that for the diffusive system, directions of the final and initial momenta are not correlated. Therefore, the first factor in Eq. (2.19) equals to

$$
\int d \boldsymbol{r} V(\boldsymbol{r}) \exp \left[\operatorname{ir}\left(\boldsymbol{p}_{\alpha}^{f}-\boldsymbol{p}_{\alpha}^{i}\right)\right] \rightarrow \overline{\tilde{V}\left(\boldsymbol{p}_{1}-\boldsymbol{p}_{2}\right)}
$$

where $\tilde{V}$ is the Fourier transform of the interaction potential and the bar denotes the averaging over the Fermi surface. Also, since the averaged density of states is translationally invariant, one can replace the integration over the interaction point $\boldsymbol{r}^{\prime \prime}$ in Eq. (2.19) by the integration over the observation point $\boldsymbol{r}$. We use the identity

$$
\int d \boldsymbol{r} A_{\alpha_{<}}^{*}\left(\boldsymbol{r}, \boldsymbol{r}^{\prime \prime} ; \epsilon\right) A_{\alpha_{>}}^{*}\left(\boldsymbol{r}^{\prime \prime}, \boldsymbol{r} ; \epsilon\right)=i t_{\alpha} A_{\alpha}^{*}\left(\boldsymbol{r}^{\prime \prime} ; \epsilon\right),
$$

which can be derived using the exact relation for the Green function. The fact that the result is proportional to the length of the trajectory $t_{\alpha}$ can be easily understood since the observation point $\boldsymbol{r}$ can be inserted anywhere along the path $\alpha$.

After the integration over $\epsilon^{\prime}$, we obtain from Eq. 2.19)

$$
\delta \nu(\epsilon) \approx \overline{\tilde{V}\left(\boldsymbol{p}_{1}-\boldsymbol{p}_{2}\right)} \operatorname{Re} \sum_{\alpha} \frac{\pi T t_{\alpha} e^{i \epsilon t_{\alpha}}}{\sinh \pi T t_{\alpha}}\left|A_{\alpha}\left(\epsilon, \boldsymbol{r}^{\prime \prime}\right)\right|^{2} .
$$

As a result, the correction to the density of states is expressed in familiar terms of the return probability, which we have already dealt with in a previous subsection! Substituting $P(t) \simeq 1 /(D t)^{d / 2}$ for the return probability, we estimate the correction to the tunneling density of states as

$$
\frac{\delta \nu(\epsilon)}{\nu} \approx \overline{\tilde{V}\left(\boldsymbol{p}_{1}-\boldsymbol{p}_{2}\right)} \int_{\tau}^{\infty} d t \cos (\epsilon t) \frac{\pi T t}{\sinh \pi T t} \frac{1}{(D t)^{d / 2}} .
$$

Therefore, the one-particle density of states $\nu(\epsilon)$ gets significantly modified due to the scattering of a given electron off the charge density oscillations of the rest of the electron gas. At low dimensions, the correction $\delta \nu(\epsilon)(2.22)$ diverges 
at large times when $T=0$ and $\epsilon=0$. One can interpret the divergence as the Bragg scattering of the incident electron off Friedel oscillations. Finite electron energy $\epsilon$ sets the upper limit for the integration because the scattering remains resonant only as long as the length of trajectory $v_{F} t$ times the difference $\hbar v_{F} / \epsilon$ between the momentum becomes of the order of unity, which corresponds to the condition $\epsilon t \lesssim \hbar$. A finite temperature $T$ also cuts the correction: it smears out the oscillating contributions related to the trajectories longer than $\hbar v_{F} / T$.

One can obtain a more familiar and convenient form 1 of (2.22) by making Fourier transform in time

$$
\frac{\delta \nu^{H}(\epsilon)}{\nu}=\operatorname{Im} \int_{0}^{\infty} \frac{d \omega}{2 \pi}\left[\tanh \left(\frac{\omega+\epsilon}{2 T}\right)+\tanh \left(\frac{\omega-\epsilon}{2 T}\right)\right] \int \frac{d^{d} Q}{(2 \pi)^{d}} \frac{2 \bar{V}}{\left(-i \omega+D Q^{2}\right)^{2}} .
$$

Here we introduced the short-hand notation $\bar{V}$ for the constant defined in Eq. (2.20).

As far as the correction to the conductivity for a short-range interaction is concerned, it is directly related to the correction to the density of states. For instance, one can determine Hartree QCC directly from Eq. (2.23)

$$
\delta \sigma^{H}=\sigma \int d \epsilon \frac{d n_{F}(\epsilon)}{d \epsilon} \frac{\delta \nu^{H}(\epsilon)}{\nu}=\frac{\sigma}{2 \pi} \operatorname{Im} \int d \omega \frac{\partial}{\partial \omega}\left(\omega \operatorname{coth} \frac{\omega}{2 T}\right) \int \frac{d^{d} Q}{(2 \pi)^{d}} \frac{2 \bar{V}}{\left(-i \omega+D Q^{2}\right)^{2}} .
$$

Inclusion of the Fock part of the self-consistent potential is also straightforward. It amounts to the addition of the Fourier transform of the interaction potential $-\tilde{V}(q=0)$ to the potential $2 \bar{V}$ in Eqs. (2.23) and (2.24). The negative sign reflects the fermionic statistics of electrons.

For the Coulomb interaction between electrons $\tilde{V}(q=0)$ diverges. This indicates that the screening should be taken into account. As a result, the correction to the tunneling density of states acquires the form

$$
\frac{\delta \nu^{C}(\epsilon)}{\nu}=-\operatorname{Im} \int_{0}^{\infty} \frac{d \omega}{2 \pi}\left[\tanh \left(\frac{\omega+\epsilon}{2 T}\right)+\tanh \left(\frac{\omega-\epsilon}{2 T}\right)\right] \int \frac{d^{d} Q}{(2 \pi)^{d}} \frac{U(Q, \omega)}{\left(-i \omega+D Q^{2}\right)^{2}},
$$

where the dynamically screened interaction potential is given by

$$
U(Q, \omega)=\left\{\frac{1}{V(Q)}+2 \nu \frac{D Q^{2}}{-i \omega+D Q^{2}}\right\}^{-1}
$$

For a long range interaction, there is no straightforward relation between the density of states and the conductivity as Eq. (2.24), since the large part of such interaction should be gauged out. One finds (see Ref. 1 and Sec. $\nabla$ of the present paper for the further technical details)

$$
\delta \sigma_{C}=-\frac{2 \sigma}{\pi \hbar d} \operatorname{Im}\left[\int d \omega \frac{\partial}{\partial \omega}\left(\omega \operatorname{coth} \frac{\omega}{2 T}\right) \int \frac{d^{d} Q}{(2 \pi)^{d}} U(Q, \omega) \frac{D Q^{2}}{\left(-i \omega+D Q^{2}\right)^{3}}\right] .
$$

For the further references, we show here the results of integration in Eq. (2.27):

$$
\begin{aligned}
\delta \sigma_{C}(T)=-\frac{e^{2}}{2 \pi^{2} \hbar} \ln \left(\frac{\hbar}{T \tau}\right) ; & d=2 ; \\
\delta \sigma_{C}(T)=-\frac{e^{2}}{\pi \hbar} \sqrt{\frac{\hbar D}{2 \pi T}}\left(\frac{3 \zeta(3 / 2)}{2}\right) ; & d=1,
\end{aligned}
$$

where $\zeta(x)$ is the Riemann zeta-function, $\zeta(3 / 2)=2.612 \ldots$.

It is crucial (and we will keep emphasizing it) that the frequency $\omega$ in Eqs. (2.25), transfered by the Coulomb interaction and (2.27) is larger than the temperature $T$. We saw that physical meaning of this correction is the elastic scattering of the electron from the potential (Hartree or Fock) created by the rest of electrons. These processes do not cause any phase breaking since they do not violate the time reversal symmetry.

\section{Energy and phase relaxation}

We turn now to discussion of the second type of effects due to the electron-electron interaction - the inelastic scattering.

In early papers (see e.g. Ref. 19) on the theory of localization, the dephasing rate $1 / \tau_{\varphi}$ was considered to be of the same order as the inelastic collision rate in clean conductors. The latter can be expressed as the sum of the electron 
- phonon $\left(1 / \tau_{e-p h} \simeq T^{3} / \Theta_{D}^{2}\right)$ and electron - electron $\left(1 / \tau_{e-e} \simeq T^{2} / E_{F}\right)$ contributions, where $E_{F}$ and $\Theta_{D}$ are the Fermi and Debye energies correspondingly (here and almost everywhere below we put $\hbar=1$ and $k_{B}=1$ ). Even under this assumption, the $e-e$ contribution dominates at low enough temperatures. It became clear later that the static disorder enhances strongly the $e-e$ contribution to the inelastic scattering rate 20 , while $1 / \tau_{e-p h}$ is less affected21. As the result, both dephasing and energy relaxation rates at low temperatures are governed by collisions between electrons.

To recall the main results on the $e-e$ dephasing rate, let us start with a single electron excitation, assuming that $T=0$, and the rest of the electron gas occupies states below the Fermi level. The dependence of $1 / \tau_{e-e}$ on the excitation energy $\epsilon$ (the energy of an electron counted from the Fermi level) can be determined in a perturbative calculation20.122. The result [Eq.(4.4) of Ref. 1] can be rewritten through the dimensionless conductance $g(L)$ (the conductance measured in units of $e^{2} / h \simeq 1 / 25.8 k \Omega$ ) of a d - dimensional cube of the size $L$

$$
\frac{1}{\tau_{e-e}(\epsilon)}=C_{d} \frac{\epsilon}{g\left(L_{\epsilon}\right)}, \quad L_{\epsilon} \equiv \sqrt{D / \epsilon}
$$

where $C_{d}$ is the dimension-and-coupling-constant-dependent coefficient. For a weakly interacting $1 d$ electron gas $C_{d}=\sqrt{2}$. Equation 2.29 ) can be also rewritten as

$$
\frac{1}{\tau_{e-e}(\epsilon)}=C_{d} \delta_{1}\left(L_{\epsilon}\right)
$$

where $\delta_{1}(L)=\left(L^{d} \nu\right)^{-1}$ is the one-particle mean level spacing in a $d$-dimensional cube of the size $L$ and $\nu$ is the one-particle density of states.

There are several interpretations of this result. One of them is based on the concept of the interaction time which becomes much longer in the disordered case due to diffusive rather than hallistic motion of electrons. It is also possible to appeal to statistical properties of exact one-electron wave functions 2324 , and we outline this interpretation below.

The inelastic rate $1 / \tau_{e-e}(\epsilon)$ is determined by a pair of collisions between electrons with all four energies - two initial $\left(\epsilon_{\alpha}\right.$ and $\left.\epsilon_{\gamma}\right)$ and two final $\left(\epsilon_{\beta}\right.$ and $\left.\epsilon_{\delta}\right)$, all the energies here are counted from the Fermi level.

Given the typical absolute alp $M_{\alpha \beta \gamma \delta}$ of the matrix element for such a collision in a sample of the size $L$, the inelastic rate can be estimated 25,23 with the help of the Fermi Golden Rule

$$
\frac{\hbar}{\tau_{e-e}} \simeq \int \frac{d \epsilon_{\beta} d \epsilon_{\gamma} d \epsilon_{\delta}}{\left[\delta_{1}(L)\right]^{3}}\left|M_{\alpha \beta \gamma \delta}\right|^{2} \delta\left(\epsilon_{\alpha}-\epsilon_{\beta}+\epsilon_{\gamma}-\epsilon_{\delta}\right)\left[1-n_{F}\left(\epsilon_{\beta}\right)\right] n_{F}\left(\epsilon_{\gamma}\right)\left[1-n_{F}\left(\epsilon_{\delta}\right)\right] .
$$

The matrix elements can be represented as integrals of products of four exact one-particle wave functions. In a disordered system, these wave functions oscillate randomly in space, and are only weakly correlated with each other. As a result, the matrix elements are random and for $L$ smaller than $L_{\epsilon}$ ( $0 d$ case), their typical absolute value $M(L)$ turns out to be of the order of $\delta_{1}(L) / g(L)$, where the small factor $g^{-1}$ reflects the weakness of the correlation between the wave functions. (In the limit $g \rightarrow \infty$ Random Matrix Theory is valid; according to this theory, there is no correlation at all between different eigenvectors and the non-diagonal matrix elements vanish.) As a result, integration in Eq. 2.31) for $0 d$ case can be easily performed 24 and for $T=0$

$$
\frac{\hbar}{\tau_{e-e}} \simeq \frac{\epsilon^{2}}{g^{2} \delta_{1}(L)}
$$

This rate increases with $\epsilon$, and at $L=L_{\epsilon}$, it becomes of the order of $\delta_{1}\left(L_{\epsilon}\right)$. This estimate corresponds exactly to Eqs. (2.29) and (2.30), and it remains valid even for large samples, $L>L_{\epsilon}$, since $1 / \tau_{e-e}$ cannot depend on $L$ in this limit.

This observation enables us to estimate the dependence of the matrix elements in a $d$-dimensional sample on the energy transfer $\omega=\epsilon_{\alpha}-\epsilon_{\beta}$, when $\omega$ is larger than the Thouless energy. Comparison of Eqs. (2.30) and (2.32) yields for this dependence

$$
\left|M_{\alpha \beta \gamma \delta}\right|^{2} \sim \frac{\delta_{1}(L)^{3} \delta_{1}\left(L_{\omega}\right)}{\omega^{2}}=\frac{\delta_{1}(L)^{4} L^{d}}{\omega^{2} L_{\omega}^{d}} \propto \omega^{-2+d / 2}, \quad \omega=\left|\epsilon_{\alpha}-\epsilon_{\beta}\right| .
$$

This energy dependence of the matrix elements reflects the properties of noninteracting disordered system and is not sensitive to the energy distribution of the electrons.

It follows from Eq. (2.33) that the matrix elements diverge when $d<4$ and $\omega \rightarrow 0$. At $T=0$ this divergence is not dangerous because of the two integrations in Eq. (2.31). However, the situation changes when the temperature is 
finite. Assuming that the energy transfer $\omega$ is much smaller than the temperature $T$, we perform the integration over $\epsilon_{\gamma}$ and $\epsilon_{\delta}$ in Eq. (2.31) and obtain with the help of Eq. (2.33)

$$
\frac{1}{\tau_{e-e}}=\int d \omega \mathcal{R}(\omega) ; \quad \mathcal{R}(\omega)=\frac{T}{\omega g\left(L_{\omega}\right)}
$$

The integral over $\omega$ dizgrges in the infrared limit for $d=1,2$. Therefore, $1 / \tau_{e-e}$ is ill-defined at finite temperatures and in low dimensions 26 .

This is not a catastrophe, though: $1 / \tau_{e-e}$ itself has no physical meaning. When the energy relaxation rate $1 / \tau_{\epsilon}$, (i.e. the inverse time of thermalization of an excitation with energy $\epsilon$ much larger than temperature $T$ ) is considered, the quasielastic processes are not important. To be more precise, for the energy diffusion the relevant quantity is

$$
\left\langle\frac{\omega^{2}}{\tau_{e-e}}\right\rangle=\int_{0}^{T} d \omega \omega^{2} \mathcal{R}(\omega)
$$

which is perfectly convergent at the lower limit. Therefore Eqs. (2.29) and (2.30) give a good estimate of $1 / \tau_{\epsilon}$.

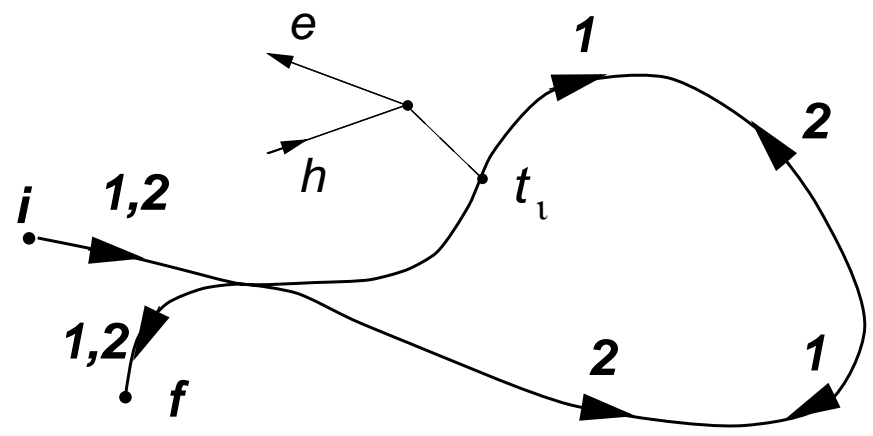

FIG. 3. (a) Example of the coherent paths involving excitation of the electron hole pair at point $t_{i}$. The phase difference between paths 1 and 2 is $\delta \phi_{12}=\omega\left(t-2 t_{i}\right)$, where $\omega$ is the energy of the excited pair.

As it was mentioned in the end of Subsection II A, the characteristic scale of the magnetic fields in the weak localization is determined by the dephasing rate $1 / \tau_{\phi}$. It turns out that this dephasing rate at low dimensions is governed by the quasielastic processes.

Let us illustrate the microscopic mechanism of the dephasing. Consider a pair of coherent paths 1 and 2 from Fig. If S. Suppose now that an electron during its motion along path 1 emits (or absorbs) an electron-hole pair with the energy $\omega$, see Fig. 3. Clearly, the interference term will be lost, unless the electron going through path 2 emits (absorbs) the same electron-hole pair. In order to evaluate, e.g., the conductivity, one has to sum up over all the final states. The contribution of a final state with the extra electron-hole pair contains the interference part from trajectories 2 and 1 with the emission of the very same pair. The only difference between paths 1 and 2 is the following: for path 1 the electron has momentum $k_{i}=k_{F}+\epsilon / v_{F}$ during the time $t_{i}$, see Fig. 3, and then it has momentum $k_{i}-\omega / v_{F}$ during the remaining time $t-t_{i}$; the electron going along path 2 has momentum $k_{i}$ during the time $t-t_{i}$ and momentum $k_{i}-\omega / v_{F}$ during time $\tau_{i}$. As a result, the phases acquired by the trajectories are no longer the same:

$$
\delta \varphi_{12}=\int \mathbf{k}_{1} d \mathbf{l}-\int \mathbf{k}_{2} d \mathbf{l}=\omega\left(t-2 t_{i}\right)
$$

Since the effect of the dephasing is significant only for $\delta \varphi_{12} \simeq 1$, the processes with the frequency, smaller than the inverse length of the path do not lead to the dephasing, and thus the relevant quantity for the path with length $t$ is not the total electron-electron relaxation rate (2.34) but rather

$$
\frac{\hbar}{\tau_{\varphi}(t)}=\int_{1 / t}^{\infty} d \omega \mathcal{R}(\omega)=\frac{T}{g(\sqrt{D t})} .
$$

It is this quantity that determines the dependence of the weak localization correction on temperature. If the magnetic field is strong enough, $\tau_{H} \lesssim \tau_{\varphi}\left(\tau_{H}\right)$, see Eqs. 2.8), the dephasing introduces a small correction of the order of $\tau_{H} / \tau_{\varphi}\left(\tau_{H}\right)$. On the other hand, if the magnetic field is weak, the length of the relevant trajectories should be determined self-consistently by putting $t=\tau_{\varphi}$ in the right-hand side of Eq. (2.36) 


$$
\frac{\hbar}{\tau_{\varphi}}=\frac{T}{g\left(\sqrt{D \tau_{\varphi}}\right)}
$$

Solution of Eq. (2.37) yields

$$
\begin{gathered}
\frac{1}{\tau_{\varphi}}=\left(\frac{e^{2} T \sqrt{\hbar D}}{\hbar \sigma_{1}}\right)^{2 / 3} ; \quad d=1, \\
\frac{1}{\tau_{\varphi}}=\frac{T}{\hbar} \frac{R_{\square} e^{2}}{2 \pi \hbar} \ln \left(\frac{\pi \hbar}{e^{2} R_{\square}}\right) ; \quad d=2,
\end{gathered}
$$

where $\sigma_{1}$ is the conductivity per unit length of a one-dimensional system (wire) and $R_{\square}$ is the sheet resistance of the two-dimensional film.

An expression for $1 / \tau_{\varphi}$ with the exact numerical coefficient (as Eqs. (2.38)) rather than an order of magnitude estimate is meaningful only if it is supplied with the explicit dependencies of the conductivity on $1 / \tau_{\varphi}$ and $H$. In order to evaluate these dependencies, one notices that the dephasing is dominated by the processes with the energy transfers much smaller than temperature. In this case, instead of considering the interaction between electrons, one can think about an electron moving in a classical electric field, created by the rest of the electron gas. Since this field fluctuates in time and space, the calculation of observable quantities involves averaging over these fluctuations. The program was realized in Ref. 27, where it was shown that the weak localization correction is still given by expression similar to Eq. (2.5)

$$
\delta \sigma_{W L}=-\frac{2 \sigma}{\pi \nu \hbar} \int_{0}^{\infty} d t\langle\mathcal{C}(\boldsymbol{r}, \boldsymbol{r} ; t .-t)\rangle_{A},
$$

where the Cooperon in the external vector potential $\boldsymbol{A}$ is given by expression similar to Eqs. (2.6) and (2.10)

$$
\left[\frac{\partial}{\partial t}+D\left(-i \boldsymbol{\nabla}_{1}-\frac{e}{c \hbar} \boldsymbol{A}\left(\boldsymbol{r}_{1}, t / 2\right)-\frac{e}{c \hbar} \boldsymbol{A}\left(\boldsymbol{r}_{1},-t / 2\right)\right)^{2}\right] \mathcal{C}\left(\boldsymbol{r}_{1}, \boldsymbol{r}_{2} ; t, t^{\prime}\right)=\delta\left(\boldsymbol{r}_{1}-\boldsymbol{r}_{2}\right) \delta\left(t-t^{\prime}\right)
$$

where $\boldsymbol{A}(\boldsymbol{r}, t)$ is the fluctuating vector potential created due to the emission and adsorption of the real electron-hole pairs. Those fields are Gaussian ones with the propagator defined as

$$
\left\langle A^{i} A^{j}\right\rangle_{Q, \omega}=-\frac{2 T}{\omega} \frac{c^{2} Q^{i} Q^{j}}{e^{2} \omega^{2}} \operatorname{Im} U(Q, \omega)=\frac{2 T}{\sigma_{d}} \frac{c^{2}}{\omega^{2}} \frac{Q^{i} Q^{j}}{Q^{2}}
$$

where $U(Q, \omega)$ is the screened interaction potential defined in Eq. (2.25), and $\sigma_{d}$ is the conductivity in $d$ dimensions. The last expansion in this formula is possible since the relevant frequencies and wavevectors are such that $Q \simeq$ $\sqrt{\omega / D} \simeq 1 / L_{\varphi} \ll 1 / a_{s}$, where $a_{s}$ is the screening radius. Formula (2.41) is consistent with Eq. (4.21) of Ref. 11. The first term in the latter formula describes the transverse fluctuation of the electromagnetic field which is usually small and we will not take them into account. Calculation described in great details in Sec. 4.2 of Ref. 1 yields

$$
\begin{array}{r}
\delta \sigma_{W L}=-\frac{e^{2}}{2 \pi^{2} \hbar}\left[\ln \frac{1}{\tau \Omega_{H}}-\Psi\left(\frac{1}{2}+\frac{1}{\tau_{\varphi} \Omega_{H}}\right)\right], \quad d=2, \quad(\boldsymbol{n} \| \boldsymbol{H}) ; \\
\delta \sigma_{W L}=-\frac{e^{2}}{2 \pi^{2} \hbar} \ln \frac{\tau_{H} \tau_{\varphi}}{\tau\left(\tau_{H}+\tau_{\phi}\right)}, \quad d=2, \quad(\boldsymbol{n} \perp \boldsymbol{H}) ; \\
\delta \sigma_{W L}=-\frac{e^{2} \sqrt{D \tau_{\varphi}}}{\pi \hbar} \frac{1}{\left[-\ln \operatorname{Ai}\left(\tau_{\varphi} / \tau_{H}\right)\right]^{\prime}}, \quad d=1,
\end{array}
$$

where the dephasing time $\tau_{\varphi}$ and the time $\tau_{H}$ associated with magnetic field are given by Eqs. (2.38) and (2.8) respectively for different dimensions $d, \operatorname{Ai}(x)$ is the Airy function, and $\Psi(x)$ is the digamma-function. Forms of Eqs. (2.42) are consistent with the general relation (2.12) 29 .

It is important to emphasize that derivation of Eqs. (2.39) - (2.41) is neither an assumption nor a phenomenological recipe - it is the result of the parametrically justified procedure. All the corrections to these formulas are small as $1 /\left(\tau_{\varphi} T\right)$. As such, this calculation is already self-contained, however some people find it useful to interpret the results appealing to Gedanken experiments similar to that of Ref. 28.

For further considerations it is useful to rewrite Eq. (2.42) in a slightly different form. First, we perform a gauge transformation of Eq. (2.40): 


$$
\left[\frac{\partial}{\partial t}+D\left(-i \nabla_{1}-\frac{2 e}{c \hbar} \boldsymbol{A}\left(\boldsymbol{r}_{1}\right)\right)^{2}+i\left(\chi\left(\boldsymbol{r}_{1}, t / 2\right)-\chi\left(\boldsymbol{r}_{1},-t / 2\right)\right)\right] \mathcal{C}\left(\boldsymbol{r}_{1}, \boldsymbol{r}_{2} ; t, t^{\prime}\right)=\delta\left(\boldsymbol{r}_{1}-\boldsymbol{r}_{2}\right) \delta\left(t-t^{\prime}\right),
$$

where $\boldsymbol{A}(\boldsymbol{r})$ represents external magnetic field, while the fluctuating electric field caused by the interaction between electrons enters through a fluctuating scalar potential $\chi(\boldsymbol{r}, t)$. According to Eq. (2.41), correlation function of this scalar potential in $(\omega, Q)$ representation equals to

$$
\left\langle\chi^{2}\right\rangle_{Q, \omega}=-\frac{2 T}{\omega} \operatorname{Im} U(Q, \omega)=\frac{2 T e^{2}}{\sigma_{d} Q^{2}} .
$$

Secondly, for the regime $\tau_{H} \ll \tau_{\varphi}$, the fluctuating potential $\chi$ can be taken into account by perturbation theory. This yields the correction to the conductivity of the form

$$
\begin{aligned}
\delta \sigma_{W L} & =-\frac{\sigma}{\pi \nu \hbar}\left[\int \frac{d^{d} Q}{(2 \pi)^{d}} \mathcal{C}(Q, 0)-\int \frac{d^{d} Q d^{d} q}{(2 \pi)^{2 d}} \int_{-T}^{T} \frac{d \omega}{2 \pi}\left(\frac{4 T e^{2}}{\sigma_{d} q^{2}}\right)\left([\mathcal{C}(Q, 0)]^{2} \mathcal{C}(\boldsymbol{Q}+\boldsymbol{q}, \omega)-|\mathcal{C}(Q, \omega)|^{2} \mathcal{C}(\boldsymbol{Q}+\boldsymbol{q}, 0)\right)\right], \\
\mathcal{C}(Q, \omega) & =\frac{1}{-i \omega+D Q^{2}+\frac{1}{\tau_{H}}}
\end{aligned}
$$

where the first term in brackets is just a weak-localization correction for non-interacting electrons and the second term is the perturbative effect of dephasing on the weak localization. Since in $d=1$ the integral over $\omega$ is determined by its lower limit, the upper limit $|\omega|=T$ is not important. In $d=2$ the integral is logarithmical and the exact definition of the cut-off is also not necessary. Integration in Eq. (2.45) gives exactly the same result as the direct expansion of Eqs. (2.42) in the small parameter $\tau_{H} / \tau_{\varphi}$. Here, and through the rest of this paper we will consider only one-dimensional wires and the films in the parallel magnetic field to avoid unnecessary technical complications.

\section{Summary of this section.}

This section is a review of the results on the effects of electron-electron interaction on the conductivity of disordered metals. We tried to give the physical interpretation to all of the rigorous formulas. The main message is that the microscopic mechanism of dephasing is the excitation or absorption of real electron-hole pairs. That is why the frequency transferred through the interaction propagator in the formulas for dephasing can not be much larger than temperature, since excitations with higher energy are forbidden by Pauli principle. As a result the dephasing rate $1 / \tau_{\phi}$ vanishes when the temperature tends to zero (see Eqs. (2.38)). The interaction correction associated with higher frequency are not due to the real processes but rather due to the scattering of the electron off the static self-consistent potential created by all the other electrons. Therefore, this scattering has nothing to do with the dephasing. In the next section, we outline "new theories" of dephasing which attempt to revise this physical picture.

\section{RECENT “THEORIES” OF ZERO-TEMPERATURE DEPHASING}

Recently, Mohanty and Webbl suggested the following expression [formula (4) of Ref. 7] for the dephasing time

$$
\frac{1}{\tau_{p}}=\frac{e^{2}}{\sigma \hbar^{2}} \int d u \int \frac{d^{d} k}{(2 \pi)^{d}} \int \frac{d \omega}{2 \pi} \hbar \omega \operatorname{coth}\left(\frac{\hbar \omega}{2 T}\right) k^{-2} \exp \left(-D k^{2}|u|-i \omega u\right) .
$$

We will denote this time $\tau_{p}$ to distinguish it from the results of previous section. We also use integration $\frac{d^{d} k}{(2 \pi)^{d}}$ instead of their $\frac{d^{2} k}{(2 \pi)^{2}}$. ( We do not quite understand why the authors of Ref. 7 conclude that "In a quasi-1D system, for any two interfering paths the wave vector $\mathbf{k}$ must be a two-dimensional vector"). Formula (3.1) was obtained as an extension of the derivation of Refs. 27, 1 in the form popularized in Ref. 30. This extension can be hardly called a derivation.

Golubev and Zaikin眴 claimed that they derived Eq. (3.1). To obtain Eq. (3.1), one needs just to substitute Eq.(74) of Ref. 9 into Eq. (71) of the same paper, see also Eqs. (7) and (8) of Ref. 8. We will discuss derivation of Ref. 9 in more details later.

Beforehand we would like to to emphasize the differences between Eq. (3.1) and the results of previous section. Though authors do not write it explicitly, the magnetic field dependence of the conductivity in Refs. 6 9 is presumably given by 


$$
\delta \sigma_{W L}=-\frac{\sigma}{\pi \nu \hbar} \int \frac{d^{d} Q}{(2 \pi)^{d}} \frac{1}{D Q^{2}+\frac{1}{\tau_{H}}+\frac{1}{\tau_{p}}}
$$

Consider the strong magnetic field $\tau_{H} \ll \tau_{p}$ limit. As it was already mentioned, in this limit one can expand Eq. (3.2) in small parameter $\tau_{H} / \tau_{p}$. Let us restrict ourselves to the first order of this expansion, substitute $\tau_{p}$ from Eq. (3.1) and integrate over $u$ in Eq. (3.1). The result is

$$
\delta \sigma_{W L}=-\frac{\sigma}{\pi \nu \hbar}\left[\int \frac{d^{d} Q}{(2 \pi)^{d}} \mathcal{C}(Q, 0)-\int \frac{d^{d} Q d^{d} q}{(2 \pi)^{2 d}} \int_{-1 / \tau}^{1 / \tau} \frac{d \omega}{2 \pi}\left(\frac{2 T e^{2}}{\sigma_{d} q^{2}}\right)\left(\hbar \omega \operatorname{coth} \frac{\hbar \omega}{2 T}\right)[\mathcal{C}(Q, 0)]^{2} \frac{1}{-i \omega+D q^{2}}\right]
$$

where $\mathcal{C}(Q, \omega)$ is defined in Eq. 2.45).

Let us now compare Eq. (3.3) with the conventional result (2.45). The first terms of both equations describe weak localization of non-interacting correction, and they simply coincide. There is no dramatic difference in the contributions to the second terms from small energy transfers. Indeed, the integrand in the second term of Eq. (3.3) at $\hbar \omega \ll T$ is of the same order of magnitude as the one in the second term of Eq. (2.45). In order to avoid an unphysical conclusion that some dephasing can be caused by the fluctuations with $\omega=0$ or $q=0$, i.e., by a static or uniform potential, the authors of Refs. 69 introduced an ad hoc infrared cut-off at $\omega \simeq 1 / \tau_{p}$.

The difference at high frequencies is much more serious. While Eq. (2.45) limits the frequency integration over the energies smaller than temperature, Eq. (3.3) extends the integration over the whole range of frequencies. As a result of this extension, the dephasing rate becomes finite at $T \rightarrow 0$. Thus, according to Eq. (3.3) the processes with the energy transfer much larger than temperature do break the time reversal symmetry and do cause dephasing. On the other hand, all the physical arguments of Sec. III apparently contradict to this statement.

We emphasize that it is extremely difficult to accept the idea of the zero-point motion dephasing from the qualitative point of view. Consider a quantum particle which moves in the environment of the harmonic oscillators: each oscillator is characterized by its frequency $\omega$. The result of the collision of the particle with an oscillator depends on the relation between $\hbar \omega$ and the temperature. If $\hbar \omega \ll T$, the oscillator is in an excited state with a high probability. The particle energy, counted from the Fermi level, is also of the order of $T$, i.e. much larger than $\hbar \omega$. As the result, the energy conservation does not prevent the energy transfer between the particle and the oscillator, i.e. the probability for the inelastic collision is substantial.

The situation for $\hbar \omega \gg T$ is quite different. Indeed, up to the exponentially small terms, the oscillator is in the ground state, and the particle has the energy smaller than $\hbar \omega$. Therefore, the energy transfer is forbidden by the energy conservation and the collision is elastic. Therefore, there is no difference whatsoever between the collision with such an oscillator and with the quenched disorder, which definitely does not dephase. As to the zero-point motion, their energy is simply the difference between the ground state energy of the oscillator and the bottom of the harmonic potential. Since, this is the energy of the ground state, it can not be absorbed, and the smearing of the wave-function of the oscillator is just a renormalization of the cross-section for the elastic processes.

However, maybe our intuition is wrong? We did not find any convincing physical argument which would require to reconsider the common understanding of dephasing in disordered systems in Refs. 60.

The only theoretical argument in favor of the zero-temperature dephasing presented up to now is the calculation of Ref. 9. We do not want to reject these calculations entirely on the basis of the qualitative picture. The history of Physics knows examples when a calculation preceded an understanding. We are going to demonstrate that it is not the case in the present situation. As we explain in the next section, the results of Golubev and Zaikin, being purely perturbative $\left(1 / \tau_{p}\right.$ is proportional to the first power of the fluctuation propagator), explicitly contradict to the straightforward calculation based on the perturbation theory.

\section{MAIN IDEA OF THE CALCULATION AND RESULTS.}

The main idea of the calculation we are about to describe is the observation that in the strong magnetic field both results of the conventional theory (2.45) and of the "new theory" (3.3) are purely perturbative in the interaction propagator $U(Q, \omega)$, see Eq. (2.25). Therefore, the truth may be revealed just by straightforward calculation of the interaction correction to the conductivity, see Fig. A . We believe that we understand the qualitative physical picture. Nevertheless, we will not use this understanding in the calculation, trying, e.g., in the very beginning to identify "diagrams relevant for inverse inelastic time $1 / \tau_{i} \ldots$ " or to introduce cut-offs in the integrations using some ideas based on the qualitative understanding. Instead, we will take into account all the diagrams of the first order in the interaction and integrate over the whole interval of the energy transfer. The only small parameter we will afford to use is the inverse dimensionless conductance of the system. 

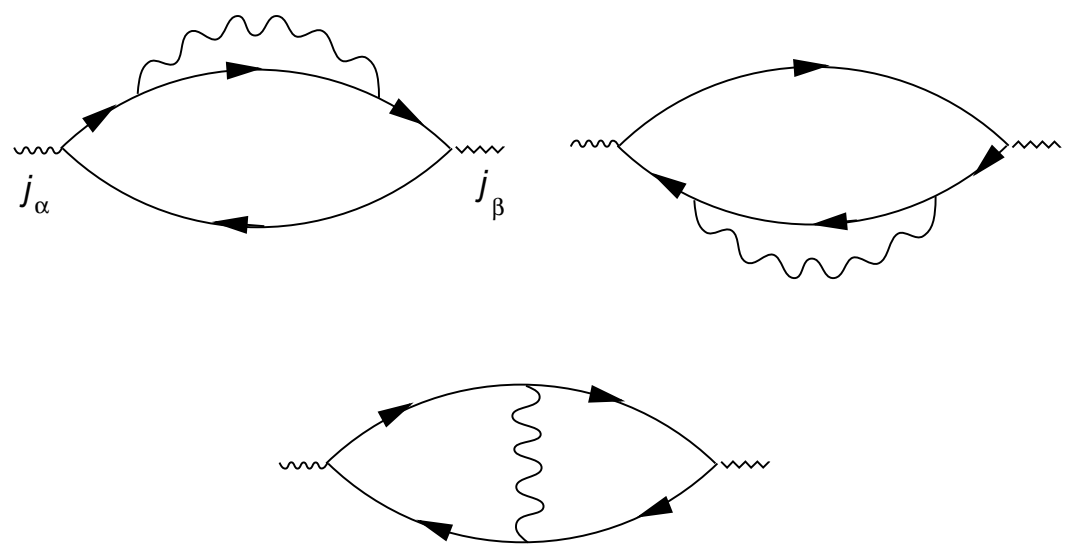

FIG. 4. Diagrams for the first order corrections to the conductivity. Electron Green functions (solid lines) are exact for the

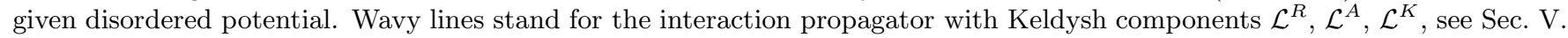

As usual, we start with expressing the correction to the conductivity through electronic and interaction propagators in a given disorder realization. This expression should be then averaged over disorder. For our purposes this averaging should be performed with taking the weak localization correction into account. It means that both zero and first order in the inverse dimensionless conductance $1 / g$ should be kept while we average. Therefore, after the averaging and integration over the energies and momenta transfers, we will obtain corrections to the conductivity which are of the first and second order in $1 / \mathrm{g}$.

It is important to emphasize that we evaluate the interaction correction to the observable (and thus gauge invariant) quantity - conductivity, rather than to a gauge noninvariant object like Cooperon.

Before we dig into details of the calculation, it is useful to realize what kind of correction we are interested in. In Sec. IIB the two kind of interaction effects - dephasing and interaction correction - were mentioned. Is it possible to separate the two corrections, when they are of the same order in $1 / g$ ? Fortunately, one can propose a procedure, which allows to perform this separation unambiguously.

Indeed, let us write down the total correction to the conductivity in the form:

$$
\delta \sigma=\delta \sigma_{W L}+\delta \sigma_{C}+\delta \sigma_{\mathrm{deph}}+\delta \sigma_{\mathrm{CWL}}+\ldots
$$

where ... stand for the corrections of the order higher than the second order in $1 / \mathrm{g}$. Here, leading weak localization and interaction corrections to the conductivity are given by, see Eqs. (2.42) and Eqs. (2.28)

$$
\begin{gathered}
\delta \sigma_{W L}=-\frac{e^{2}}{2 \pi^{2} \hbar} \ln \frac{1}{\tau \Omega_{H}} ; \quad \delta \sigma_{C}(T)=-\frac{e^{2}}{2 \pi^{2} \hbar} \ln \left(\frac{\hbar}{T \tau}\right) ; \quad d=2 ; \\
\delta \sigma_{W L}=-\frac{e^{2} \sqrt{D \tau_{H}}}{\pi \hbar} ; \quad \delta \sigma_{C}(T)=-\frac{e^{2}}{\pi \hbar} \sqrt{\frac{\hbar D}{2 \pi T}}\left(\frac{3 \zeta(3 / 2)}{2}\right) ; \quad d=1,
\end{gathered}
$$

and time $\tau_{H} \gg \hbar / T$ is given by Eqs. (2.8). The term $\sigma_{\text {deph }}$ appears as the first order correction to the time scale cutting off the weak localization correction. In accordance with the previous discussion, it should have the form

$$
\begin{gathered}
\delta \sigma_{\mathrm{deph}}=\frac{e^{2}}{2 \pi^{2} \hbar} \frac{\tau_{H}}{\tau_{\varphi}} ; \quad d=2 ; \\
\delta \sigma_{\mathrm{deph}}=\frac{e^{2} \sqrt{D \tau_{H}}}{\pi \hbar} \frac{\tau_{H}}{\tau_{\varphi}} ; \quad d=1,
\end{gathered}
$$

where the scale $1 / \tau_{\varphi}$ is to be determined. If the calculations of Golubev and Zaikin were correct, this scale would coincide with that given by Eqs. (77) and (81) of Ref. 9 .

The last term in Eq. (4.1) characterizes the effect of weak localization on the interaction correction and vice versa, which do not break the time reversal invariance. On purely dimensional grounds they can be written as

$$
\begin{gathered}
\delta \sigma_{\mathrm{CWL}}=\frac{e^{2}}{2 \pi^{2} \hbar} \frac{R_{\square} e^{2}}{2 \pi^{2} \hbar}\left(\alpha_{1} \ln ^{2} T \tau+\alpha_{2} \ln ^{2} \Omega_{H} \tau+\alpha_{3} \ln T \tau \ln \Omega_{H} \tau\right) ; \quad d=2 ; \\
\delta \sigma_{\mathrm{CWL}}=\alpha \frac{e^{2} \sqrt{D \tau_{H}}}{\pi \hbar}\left(\frac{e^{2}}{\pi \hbar \sigma} \sqrt{\frac{\hbar D}{T}}\right) ; \quad d=1,
\end{gathered}
$$


where $\sigma_{1}$ is the conductivity per unit length of a one-dimensional system (wire), $R_{\square}$ is the sheet resistance of the two-dimensional film, and $\alpha_{i}$ are numerical coefficients to be found. One can separate contributions (4.3) and (4.4) using the apparent difference in the magnetic field dependence of these two terms.

Now it is time to outline our strategy. We calculate the correction to the conductivity in the first order in fluctuation propagator. Next step is to average the result over disorder, taking weak localization into account in each term. Finally, we separate the result into terms of the form (4.3) and (4.4), thus evaluating the dephasing rate $1 / \tau_{\varphi}$.

This program is described in details in Sec. V] but we would like to present and discuss the results right now:

1. All the terms which scale according to Eq. (4.3) are produced by the expression of the form similar to Eq. (2.45)

$$
\delta \sigma_{\mathrm{deph}}=\frac{\sigma}{\pi \nu \hbar} \int \frac{d^{d} Q d^{d} q}{(2 \pi)^{2 d}} \int \frac{d \omega}{2 \pi} f\left(\frac{\omega}{T}\right)\left(\frac{4 T e^{2}}{\sigma_{d} q^{2}}\right)\left([\mathcal{C}(Q, 0)]^{2} \mathcal{C}(\boldsymbol{Q}+\boldsymbol{q}, \omega)-|\mathcal{C}(Q, \omega)|^{2} \mathcal{C}(\boldsymbol{Q}+\boldsymbol{q}, 0)\right)
$$

where Cooperon $\mathcal{C}$ is defined by Eq. (2.45) and dimensionless function $f$ is given by

$$
f\left(\frac{\omega}{T}\right)=\left(\frac{\omega}{4 T}\right) \int d \epsilon\left(\frac{d}{d \epsilon} \tanh \frac{\epsilon}{2 T}\right)\left(\operatorname{coth} \frac{\omega}{2 T}+\tanh \frac{\epsilon-\omega}{2 T}\right)=\left(\frac{\omega}{2 T}\right)^{2} \frac{1}{\sinh ^{2} \frac{\omega}{2 T}} .
$$

2. For the cross term of interaction and weak localization of the type $(4.4)$ we found

$$
\delta \sigma_{C W L}=\frac{e^{2}}{\pi^{2} \hbar} \frac{e^{2}}{\pi^{2} \hbar \sigma_{d}}\left(\frac{T}{\hbar D}\right)^{d-2} \frac{1}{2^{d}}\left[\left(2 \pi T \tau_{H}\right)^{1-d / 2} \frac{(4-d)}{d} \Gamma^{2}(2-d / 2) \zeta(2-d / 2)-\frac{2}{2-d} \zeta(3-d)\right],
$$

where $\Gamma(x)$ is the Gamma function and $\zeta(x)$ is the Riemann zeta-function. Here, we retained only most singular at $d \rightarrow 2$ contribution into the second not dependent on magnetic field term.

Note, that in Eq. (4.7) the ultraviolet convergence is provided by the function $f(\omega / T)$ from Eq. (4.6), which falls down exponentially at $\omega \gg T$ (rather than by the limit $\simeq T$ as in Eq. (2.45)). The structure of $f(\omega / T)$ deserves some discussion. The coth term is the only term which was taken into account in Ref. 7 g. This term indeed dominates for $\omega \ll T$. At high frequencies, however, this term is canceled out almost exactly by the second tanh term 31 . It is this term, neglected in Ref. 7 9 , that is responsible for the Pauli principle. The clearly indicates the inelastic origin $\tau_{\phi}$ in accord with the arguments of Sec. III. The reason why the authors of Ref. 8.9 missed this term is, as it is explained in Sec. VI, in the incorrect procedure of the disorder average.

Since Eq. (4.5) is essentially the same as Eq. 2.45) in the frequency domain $\omega \ll T$, which gives the main contribution in low dimensional systems, this is not surprising that the results of the integration are perfectly consistent with Refs. 1. 27. In the leading order in $T \tau_{H}$, we found

$$
\begin{aligned}
\delta \sigma_{\text {deph }} & =\frac{e^{2}}{2 \pi^{2} \hbar} \frac{T \tau_{H}}{\hbar} \frac{R_{\square} e^{2}}{2 \pi \hbar} \ln \left(\frac{T \tau_{H}}{\hbar}\right) ; \quad d=2 ; \\
\delta \sigma_{\text {deph }} & =\frac{e^{2} \sqrt{D \tau_{H}}}{\pi \hbar} \tau_{H}^{3 / 2} D^{1 / 2} \frac{e^{2} T}{4 \hbar^{2} \sigma_{1}} ; \quad d=1,
\end{aligned}
$$

which according to Eq. (4.3) means that

$$
\begin{gathered}
\frac{1}{\tau_{\varphi}}=\frac{T}{\hbar} \frac{R_{\square} e^{2}}{2 \pi \hbar} \ln \left(\frac{T \tau_{H}}{\hbar}\right) ; \quad d=2 ; \\
\frac{1}{\tau_{\varphi}}=\frac{T}{4 \hbar} \frac{e^{2} \sqrt{D \tau_{H}}}{\hbar \sigma_{1}} ; \quad d=1 .
\end{gathered}
$$

Equations (4.9) are applicable provided that $\tau_{H} \ll \tau_{\varphi}$; in the opposite case $1 / \tau_{H}$ in the right-hand side of these equations, should be substituted by $1 / \tau_{\varphi}$ and the procedure of Ref. 27 is required for finding the temperature dependence for the magnetoresistance.

The cross term of weak localization and interaction (4.7) is smaller than the dephasing term. We obtain for a one dimensional case from Eq. (4.7)

$$
\delta \sigma_{\mathrm{CWL}}=\frac{e^{2} \sqrt{D \tau_{H}}}{2 \pi \hbar}\left(\frac{e^{2}}{2 \pi \hbar \sigma} \sqrt{\frac{\hbar D}{2 \pi T}}\right) 3 \zeta(3 / 2) ; \quad d=1,
$$


where $\sigma_{1}$ is the conductivity per unit length of one-dimensional system (wire), and $\zeta(x)$ is the Riemann zeta-function, $\zeta(3 / 2)=2.612 \ldots$

Smallness of the term $(4.10)$ in comparison with the dephasing term $(4.9)$ one more time illustrates the simple fact that the procedure of Ref. 27 is the parametrically justified program of summation of most divergent term in each order of perturbation theory in the interaction.

The resulting conductivity is given by the sum of Eq. (4.5) and Eq. (4.7). However, keeping the dephasing term in the leading approximation (4.8) and taking into account the correction (4.7) would be overstepping the accuracy of the approximation, since the contribution from $\omega \simeq T$ in Eq. (4.5) is of the same order as Eq. (4.7). Therefore, the calculation of the integral in Eq. (4.5) should be performed with the same accuracy as Eq. (4.7). It gives

$$
\begin{aligned}
\delta \sigma_{d e p h} & =\frac{e^{2}}{\pi^{2} \hbar} \frac{e^{2}}{\pi \hbar \sigma_{d}} \frac{4 \Gamma^{2}(2-d / 2)}{2^{d}(2-d)} \\
& \times\left\{\left[\frac{T \tau_{H}}{\hbar}\right]\left[\frac{1}{2(3-d)}\left(\frac{1}{2 \pi D \tau_{H}}\right)^{d-2}+\left(\frac{d \cos \frac{\pi d}{4}}{2 \pi}\right) \zeta\left(\frac{d}{2}\right) \Gamma\left(\frac{d}{2}\right)\left(\frac{1}{2 \pi D \tau_{H}}\right)^{d / 2-1}\left(\frac{T}{2 \pi \hbar D}\right)^{d / 2-1}\right]\right. \\
& \left.+\frac{1}{2 \pi}\left(\frac{T}{\hbar D}\right)^{d-2}\left[\zeta\left(2-\frac{d}{2}\right) \frac{(2-d)(3-d)}{4}\left(2 \pi T \tau_{H}\right)^{1-d / 2}+\frac{2 d}{6-d} \frac{\Gamma(3-d) \Gamma(d / 2)}{\Gamma(2-d / 2)} \zeta(3-d)\right]\right\} .
\end{aligned}
$$

Summing Eq. (4.7) and Eq. (4.11), we obtain the final result for the overall effect of the electron-electron interaction on the weak localization correction

$$
\begin{aligned}
\delta \sigma_{I \times W L} & =\frac{e^{2}}{\pi^{2} \hbar} \frac{e^{2}}{\pi \hbar \sigma_{d}} \frac{4 \Gamma^{2}(2-d / 2)}{2^{d}} \\
& \times\left\{\frac{1}{2-d}\left[\frac{T \tau_{H}}{\hbar}\right]\left[\frac{1}{2(3-d)}\left(\frac{1}{2 \pi D \tau_{H}}\right)^{d-2}+\left(\frac{d \cos \frac{\pi d}{4}}{2 \pi}\right) \zeta\left(\frac{d}{2}\right) \Gamma\left(\frac{d}{2}\right)\left(\frac{1}{2 \pi D \tau_{H}}\right)^{d / 2-1}\left(\frac{T}{2 \pi \hbar D}\right)^{d / 2-1}\right]\right. \\
& \left.+\frac{1}{2 \pi}\left(\frac{T}{\hbar D}\right)^{d-2}\left[\zeta\left(2-\frac{d}{2}\right) \frac{8+d-d^{2}}{4 d}\left(2 \pi T \tau_{H}\right)^{1-d / 2}+\frac{1}{2-d} \mathcal{O}(1)\right]\right\}
\end{aligned}
$$

Taking the last term in brackets into account would be beyond the accuracy of the theory since the second order interaction correction gives the similar contribution.

Let us write down the final results for the correction (4.12) in different dimensionalities

$$
\begin{aligned}
& \delta \sigma_{I \times W L}=\frac{e^{2}}{\pi \hbar} \frac{e^{2}}{\hbar \sigma_{d}}\left\{D \tau_{H}\left(\frac{T \tau_{H}}{4 \hbar}\right)\left[1+\zeta\left(\frac{1}{2}\right) \sqrt{\frac{2 \hbar}{\pi T \tau_{H}}}\right]+\frac{\zeta\left(\frac{3}{2}\right)}{\pi} \sqrt{\frac{\hbar D^{2} \tau_{H}}{2 \pi T}}\right\}, \quad d=1, \\
& \delta \sigma_{I \times W L}=\frac{e^{2}}{2 \pi^{2} \hbar} \frac{R_{\square} e^{2}}{2 \pi^{2} \hbar}\left\{\frac{\pi T \tau_{H}}{\hbar}\left[\ln \left(\frac{T \tau_{H}}{\hbar}\right)+1\right]+\frac{3}{2} \ln \left(\frac{\tau_{H}}{\tau}\right)+\mathcal{O}[\ln (T \tau / \hbar)]\right\}, \quad d=2
\end{aligned}
$$

where $\zeta(1 / 2)=-1.461 \ldots, \zeta(3 / 2)=2.612 \ldots$..

To conclude, the results of the well-controlled perturbation theory contradict to those of Ref. 9. Thus, the statement made in this paper that the "analysis should in principle include all diagrams" is false. The theoretical conclusion about saturation of the dephasing rate at $T \rightarrow 0$ contradicts not only the physical intuition but also the straightforward calculation.

\section{CALCULATION OF THE INTERACTION CORRECTION TO THE CONDUCTIVITY}

\section{A. Conductivity for a given disorder realization}

In this section we will use units $\hbar=c=1$. We start with the conventional expression for the current density $\boldsymbol{j}(\boldsymbol{r}, t)$ in terms of the electron Green functions in Keldysh technique 32

$$
\boldsymbol{j}(\boldsymbol{r}, t)=-\frac{1}{4 m} \operatorname{Tr}^{K}\left(\hat{\tau}_{2}-2 \hat{\tau}_{1}\right)\left[\boldsymbol{\nabla} \boldsymbol{r}_{1}-\nabla \boldsymbol{r}_{2}-2 i e \boldsymbol{A}(\boldsymbol{r})\right] \hat{G}\left(\boldsymbol{r}_{1}, t_{1} ; \boldsymbol{r}_{2}, t_{2}\right) \mid \begin{gathered}
\boldsymbol{r}_{1}=\boldsymbol{r}_{2} \\
t_{1}=t_{2}+0=t
\end{gathered}
$$


where the trace is performed in the Keldysh space, the matrix Green function is defined as

$$
\hat{G}(1,2)=\left(\begin{array}{cc}
G^{R}(1,2) & G^{K}(1,2) \\
0 & G^{A}(1,2)
\end{array}\right)
$$

and the Pauli matrices in the Keldysh space are

$$
\hat{\tau}_{1}=\left(\begin{array}{ll}
1 & 0 \\
0 & 1
\end{array}\right), \quad \hat{\tau}_{2}=\left(\begin{array}{ll}
0 & 1 \\
1 & 0
\end{array}\right) .
$$

Entrees in Eq. (5.2) are given by

$$
\begin{array}{r}
i G^{R}(1,2)=\theta\left(t_{1}-t_{2}\right)\left\langle\hat{\psi}(1) \hat{\psi}^{\dagger}(2)+\hat{\psi}^{\dagger}(2) \hat{\psi}(1)\right\rangle \\
i G^{A}(1,2)=-\theta\left(t_{2}-t_{1}\right)\left\langle\hat{\psi}(1) \hat{\psi}^{\dagger}(2)+\hat{\psi}^{\dagger}(2) \hat{\psi}(1)\right\rangle \\
i G^{K}(1,2)=\left\langle\hat{\psi}(1) \hat{\psi}^{\dagger}(2)-\hat{\psi}^{\dagger}(2) \hat{\psi}(1)\right\rangle
\end{array}
$$

where $\langle\ldots\rangle$ means averaging over quantum mechanical states (do not confuse with the ensemble averaging !), 1 is the short hand notation for $\left(\boldsymbol{r}_{1}, t_{1}\right), \hat{\psi}(\boldsymbol{r}, t)$ is the fermionic operator in the Heisenberg representation, and $\theta(x)$ is the step function. Spin indices are omitted and summation over them is implied whenever it is necessary.

Let us now expand the Green function up to the first order in the interaction propagator

$$
\hat{\mathcal{L}}(1,2)=\left(\begin{array}{cc}
\mathcal{L}^{K}(1,2) & \mathcal{L}^{R}(1,2) \\
\mathcal{L}^{A}(1,2) & 0
\end{array}\right) .
$$

In the short-hand notation $\int d 3 \equiv \int d t_{3} d \boldsymbol{r}_{3}$ the connection between Green functions in the presence $(\hat{G})$ and in the absence $\left(\hat{G}^{(n)}\right)$ of the interaction can be written as

$$
i \hat{G}(1,2)=i \hat{G}^{(n)}(1,2)+\frac{i^{3}}{2} \int d 3 \int d 4 \sum_{i, j=1,2} \hat{G}^{(n)}(1,3) \hat{\tau}_{i} \hat{G}^{(n)}(3,4) \hat{\tau}_{j} \hat{G}^{(n)}(4,2) \mathcal{L}_{i j}(3,4),
$$

For our purposes it will be sufficient to use the equilibrium values of the matrix elements of the interaction propagator $\hat{\mathcal{L}}(1,2)$. In this case these matrix elements do not depend on $t_{1}+t_{2}$. Performing Fourier transform in the difference of the times $t_{-}=t_{1}-t_{2}$

$$
\hat{\mathcal{L}}(\omega)=\int d t_{-} \hat{\mathcal{L}}\left(t_{-}\right) \exp \left(i \omega t_{-}\right)
$$

one obtains the following connection between the matrix elements 32

$$
\mathcal{L}^{K}(\omega)=\operatorname{coth} \frac{\omega}{2 T}\left[\mathcal{L}^{R}(\omega)-\mathcal{L}^{A}(\omega)\right] .
$$

In the leading order, the retarded and advanced parts of the interaction propagator depend on only on the difference of its coordinates. For the Coulomb interaction they are related to the screened interaction potential by

$$
\mathcal{L}^{R}(\omega, Q)=\mathcal{L}^{A}(-\omega, Q)=-U(\omega, Q) \approx-\frac{1}{2 \nu}\left[\frac{-i \omega+D(\omega) Q^{2}}{D(\omega) Q^{2}}\right] .
$$

Here $\nu$ is the density of states per one spin and $U(\omega, Q)$ is the dynamically screened interaction potential (2.26). To calculate the cross-term $\delta \sigma_{C W L}$ correctly, one has to include the weak localization correction to the diffusion coefficient $D$ (see below). This is the reason why the diffusion coefficient $D(\omega)$ acquired the frequency dependence. The mesoscopic fluctuations of the propagator $\hat{\mathcal{L}}(\omega)$ are disregarded in the present calculation, since they lead to the higher order in $1 / g$ corrections to the conductivity.

Now, we substitute Eq. (5.6) into Eq. (5.1). In the equilibrium, the longitudinal current vanishes, and therefore all the Green functions should be expanded up to the first order in the external vector potential $\boldsymbol{A}^{\text {ext }}$ which represents the applied electric field $\boldsymbol{E}=\partial_{t} \boldsymbol{A}^{\text {ext }}$ :

$$
\hat{G}^{(n)}(1,2)=\hat{G}^{(0)}(1,2)+\int d 3 \hat{G}^{(0)}(1,3) \hat{\boldsymbol{j}} \boldsymbol{A}^{\mathrm{ext}}\left(t_{3}\right) \hat{G}^{(0)}(3,2) .
$$


The current operator in the coordinate representation is defined as

$$
f_{1}(\boldsymbol{r}) \hat{\boldsymbol{j}} f_{2}(\boldsymbol{r})=\frac{i e}{2 m}\left[\left(\boldsymbol{\nabla} f_{1}\right) f_{2}-f_{1} \boldsymbol{\nabla} f_{2}\right]-\frac{e \boldsymbol{A}(\boldsymbol{r})}{m} f_{1}(\boldsymbol{r}) f_{2}(\boldsymbol{r})
$$

for any two functions $f_{1,2}$.

The time Fourier transform of the matrix elements of equilibrium Green functions $\hat{G}^{(0)}$ in Eq. (5.9) are given by

$$
\begin{array}{r}
G^{K}\left(\epsilon ; \boldsymbol{r}_{1}, \boldsymbol{r}_{2}\right)=\tanh \frac{\epsilon}{2 T}\left[G^{R}\left(\epsilon ; \boldsymbol{r}_{1}, \boldsymbol{r}_{2}\right)-G^{A}\left(\epsilon ; \boldsymbol{r}_{1}, \boldsymbol{r}_{2}\right)\right] \\
G^{R}\left(\epsilon ; \boldsymbol{r}_{1}, \boldsymbol{r}_{2}\right)=\frac{1}{\epsilon+\mu-\hat{H}+i 0}, \quad G^{A}\left(\epsilon ; \boldsymbol{r}_{1}, \boldsymbol{r}_{2}\right)=\frac{1}{\epsilon+\mu-\hat{H}-i 0},
\end{array}
$$

where $\hat{H}$ is the exact one-electron Hamiltonian for a given system, and $\mu$ is the chemical potential. In Eq. (5.11) and in all subsequent formulas we will omit superscript (0) since only such equilibrium Green functions appear in all further calculations.

Substituting Eqs. (5.9) into Eq. (5.6) and the result into Eq. (5.1) we obtain after simple algebra the Ohm law $\boldsymbol{j}=\hat{\sigma} \boldsymbol{E}$ with the conductivity tensor

$$
\begin{aligned}
& \sigma_{\alpha \beta}=\sigma_{\alpha \beta}^{\mathrm{ni}}+\delta \sigma_{\alpha \beta}^{\mathrm{deph}}+\delta \sigma_{\alpha \beta}^{\mathrm{int}} \\
& \sigma_{\alpha \beta}^{\mathrm{ni}}=\int \frac{d \boldsymbol{r}_{1} d \boldsymbol{r}_{2}}{\mathcal{V}} \int \frac{d \epsilon}{8 \pi}\left(\frac{d}{d \epsilon} \tanh \frac{\epsilon}{2 T}\right)\left[G_{12}^{R}(\epsilon)-G_{12}^{A}(\epsilon)\right] \hat{j}_{\alpha}\left[G_{21}^{A}(\epsilon)-G_{21}^{R}(\epsilon)\right] \hat{j}_{\beta} \\
& \sigma_{\alpha \beta}^{\mathrm{deph}}=-\frac{i}{16} \int \frac{d \boldsymbol{r}_{1} d \boldsymbol{r}_{2} d \boldsymbol{r}_{3} d \boldsymbol{r}_{4}}{\mathcal{V}} \int \frac{d \epsilon}{2 \pi} \frac{d \omega}{2 \pi}\left(\frac{d}{d \epsilon} \tanh \frac{\epsilon}{2 T}\right)\left(\operatorname{coth} \frac{\omega}{2 T}+\tanh \frac{\epsilon-\omega}{2 T}\right)\left[\mathcal{L}_{34}^{R}(\omega)-\mathcal{L}_{34}^{A}(\omega)\right] \times \\
& \quad\left\{2 \hat{j}_{\alpha}\left[G_{12}^{R}(\epsilon)-G_{12}^{A}(\epsilon)\right] \hat{j}_{\beta}\left[G_{23}^{A}(\epsilon) G_{34}^{A}(\epsilon-\omega) G_{41}^{A}(\epsilon)-G_{23}^{R}(\epsilon) G_{34}^{R}(\epsilon-\omega) G_{41}^{R}(\epsilon)\right]\right. \\
& \left.\quad+\hat{j}_{\alpha}\left[G_{13}^{R}(\epsilon) G_{32}^{R}(\epsilon-\omega)-G_{13}^{A}(\epsilon) G_{32}^{A}(\epsilon-\omega)\right] \hat{j}_{\beta}\left[G_{24}^{A}(\epsilon-\omega) G_{41}^{A}(\epsilon)-G_{24}^{R}(\epsilon-\omega) G_{41}^{R}(\epsilon)\right]+\alpha \leftrightarrow \beta\right\} \\
& \sigma_{\alpha \beta}^{\mathrm{int}}=-\frac{i}{8} \int \frac{d \boldsymbol{r}_{1} d \boldsymbol{r}_{2} d \boldsymbol{r}_{3} d \boldsymbol{r}_{4}}{\mathcal{V}} \int \frac{d \epsilon}{2 \pi} \frac{d \omega}{2 \pi}\left(\frac{d}{d \epsilon} \tanh \frac{\epsilon}{2 T}\right) \tanh \frac{\epsilon-\omega}{2 T} \times \\
& \quad\left\{\hat{j}_{\alpha}\left[G_{12}^{R}(\epsilon)-G_{12}^{A}(\epsilon)\right] \hat{j}_{\beta}\left[G_{23}^{A}(\epsilon) G_{41}^{A}(\epsilon)-G_{23}^{R}(\epsilon) G_{41}^{R}(\epsilon)\right]\left[G_{34}^{R}(\epsilon-\omega) \mathcal{L}_{34}^{A}(\omega)-G_{34}^{A}(\epsilon-\omega) \mathcal{L}_{34}^{R}(\omega)\right]\right. \\
& \quad+\left[G_{13}^{R}(\epsilon)-G_{13}^{A}(\epsilon)\right] \hat{j}_{\alpha}\left[G_{14}^{A}(\epsilon)-G_{14}^{R}(\epsilon)\right]\left[G_{42}^{R}(\epsilon-\omega) \hat{j}_{\beta} G_{23}^{R}(\epsilon-\omega) \mathcal{L}_{34}^{A}(\omega)-G_{42}^{A}(\epsilon-\omega) \hat{j}_{\beta} G_{23}^{A}(\epsilon-\omega) \mathcal{L}_{34}^{R}(\omega)\right] \\
& \quad+\alpha \leftrightarrow \beta\}
\end{aligned}
$$

where $\mathcal{V}$ is the volume of the system, $\hat{j}_{\alpha}$ is the component of current operator defined in Eq. (5.10), and the short-hand notation $G_{i j}(\epsilon) \equiv G\left(\epsilon ; \boldsymbol{r}_{i}, \boldsymbol{r}_{j}\right)$ is introduced. In Eqs. (5.12b) - (5.12d) only the symmetric part of the conductivity tensor is retained. We emphasize that all these formulas are exact in the first order of perturbation theory, i.e. we made no assumptions about the behavior of the Green functions or the relevant frequencies in the interaction propagator. At this stage separation of the interaction correction into two pieces $(5.12 \mathrm{c})$ and $(5.12 \mathrm{~d})$ is just a matter of convenience physical meaning of which will become clear shortly.

Equations (5.12), which express the conductivity in terms of the exact retarded and advance Green functions for a given realization of potential, are the main results of this subsection. In order to find the physical conductivity of a large sample, one has to perform the disorder averaging. This is the subject of the following subsection.

\section{B. Averaging over disorder}

Let us start the disorder averaging of Eqs. $(5.12 \mathrm{~b})-(5.12 \mathrm{~d})$ with the observation that any mean product of the electron Green functions does not depend on their common energy $\epsilon$. This fact allows to perform the energy integration in Eqs. (5.12b $)$ - (5.12d $)$. It is also important and useful to notice that after the averaging, the products containing only retarded or only advanced Green functions vanish. Using this feature, we simplify Eqs. (5.12) to the form

$$
\sigma_{\alpha \beta}^{\mathrm{ni}}=\frac{1}{4 \pi} \int \frac{d \boldsymbol{r}_{1} d \boldsymbol{r}_{2}}{\mathcal{V}}\left\langle\left\langle G_{12}^{R}(\epsilon) \hat{j}_{\alpha} G_{21}^{A}(\epsilon) \hat{j}_{\beta}\right\rangle\right\rangle+\alpha \leftrightarrow \beta
$$




$$
\begin{aligned}
& \sigma_{\alpha \beta}^{\mathrm{deph}}=-\frac{i}{8 \pi} \int \frac{d \boldsymbol{r}_{1} d \boldsymbol{r}_{2} d \boldsymbol{r}_{3} d \boldsymbol{r}_{4}}{\mathcal{V}} \int \frac{d \omega}{2 \pi}\left(\frac{\omega}{2 T \sinh ^{2} \frac{\omega}{2 T}}\right)\left[\mathcal{L}_{34}^{R}(\omega)-\mathcal{L}_{34}^{A}(\omega)\right] \times \\
& \left\{\left\langle\left\langle\hat{j}_{\alpha} G_{12}^{R}(\epsilon) \hat{j}_{\beta} G_{23}^{A}(\epsilon) G_{34}^{A}(\epsilon-\omega) G_{41}^{A}(\epsilon)\right\rangle\right\rangle+\left\langle\left\langle\hat{j}_{\alpha} G_{12}^{A}(\epsilon) \hat{j}_{\beta} G_{23}^{R}(\epsilon) G_{34}^{R}(\epsilon-\omega) G_{41}^{R}(\epsilon)\right\rangle\right\rangle\right. \\
& \left.\quad+\left\langle\left\langle\hat{j}_{\alpha} G_{13}^{R}(\epsilon) G_{32}^{R}(\epsilon-\omega) \hat{j}_{\beta} G_{24}^{A}(\epsilon-\omega) G_{41}^{A}(\epsilon)\right\rangle\right\rangle+\alpha \leftrightarrow \beta\right\} ; \\
& \sigma_{\alpha \beta}^{\mathrm{int}}=-\frac{1}{4 \pi} \operatorname{Im} \int \frac{d \boldsymbol{r}_{1} d \boldsymbol{r}_{2} d \boldsymbol{r}_{3} d \boldsymbol{r}_{4}}{\mathcal{V}} \int \frac{d \omega}{2 \pi}\left[\frac{d}{d \omega}\left(\omega \operatorname{coth} \frac{\omega}{2 T}\right)\right] \mathcal{L}_{34}^{A}(\omega) \times \\
& \left\{\left\langle\left\langle\hat{j}_{\alpha} G_{12}^{A}(\epsilon) \hat{j}_{\beta} G_{23}^{R}(\epsilon) G_{34}^{R}(\epsilon-\omega) G_{41}^{R}(\epsilon)\right\rangle\right\rangle+\left\langle\left\langle\hat{j}_{\alpha} G_{12}^{R}(\epsilon) \hat{j}_{\beta} G_{23}^{A}(\epsilon) G_{34}^{R}(\epsilon-\omega) G_{41}^{A}(\epsilon)\right\rangle\right\rangle\right. \\
& -\left\langle\left\langle G_{31}^{A}(\epsilon) \hat{j}_{\alpha} G_{12}^{A}(\epsilon) \hat{j}_{\beta} G_{24}^{A}(\epsilon) G_{43}^{R}(\epsilon-\omega)\right\rangle\right\rangle-\left\langle\left\langle G_{31}^{A}(\epsilon) \hat{j}_{\alpha} G_{14}^{A}(\epsilon) G_{42}^{R}(\epsilon-\omega) \hat{j}_{\beta} G_{23}^{R}(\epsilon-\omega)\right\rangle\right\rangle \\
& \left.+2\left\langle\left\langle G_{31}^{R}(\epsilon) \hat{j}_{\alpha} G_{14}^{A}(\epsilon) G_{42}^{R}(\epsilon-\omega) \hat{j}_{\beta} G_{23}^{R}(\epsilon-\omega)\right\rangle\right\rangle+\alpha \leftrightarrow \beta\right\}
\end{aligned}
$$

where $\langle\langle\ldots\rangle\rangle$ stand for the disorder average.

Equations (5.13) express the average conductance in terms of the average of products of Green functions of a noninteracting system. Those averages should be performed with taking the weak localization correction into account. We will perform the average using the diagrammatic techniquel 33 ; the same results can be obtained with the help of supersymmetric non-linear $\sigma$-model 34 .

Evaluation of the mean conductivity of non-interacting electrons $\sigma^{n i}$, i.e., averaging of the equation (5.13a), is now a textbook problem. Summation of the diagrams in Fig. 5 gives $\sigma_{\alpha \beta}=\delta_{\alpha \beta}\left(\sigma+\delta \sigma_{W L}\right)$ where Drude conductivity $\sigma$ and the weak localization correction given by (1.1) and the weak localization correction $\delta \sigma_{W L}$ are given by (1.1) and (2.5) respectively.

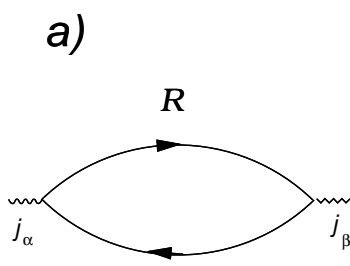

A

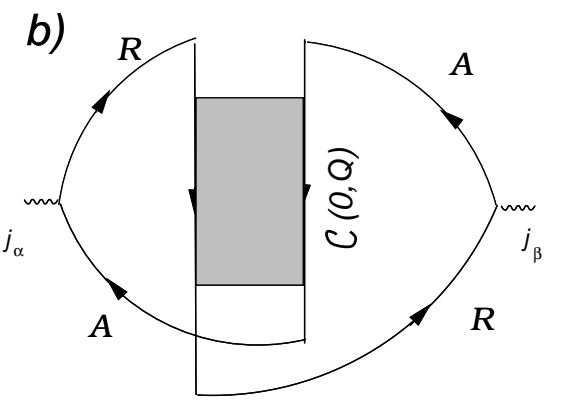

c)
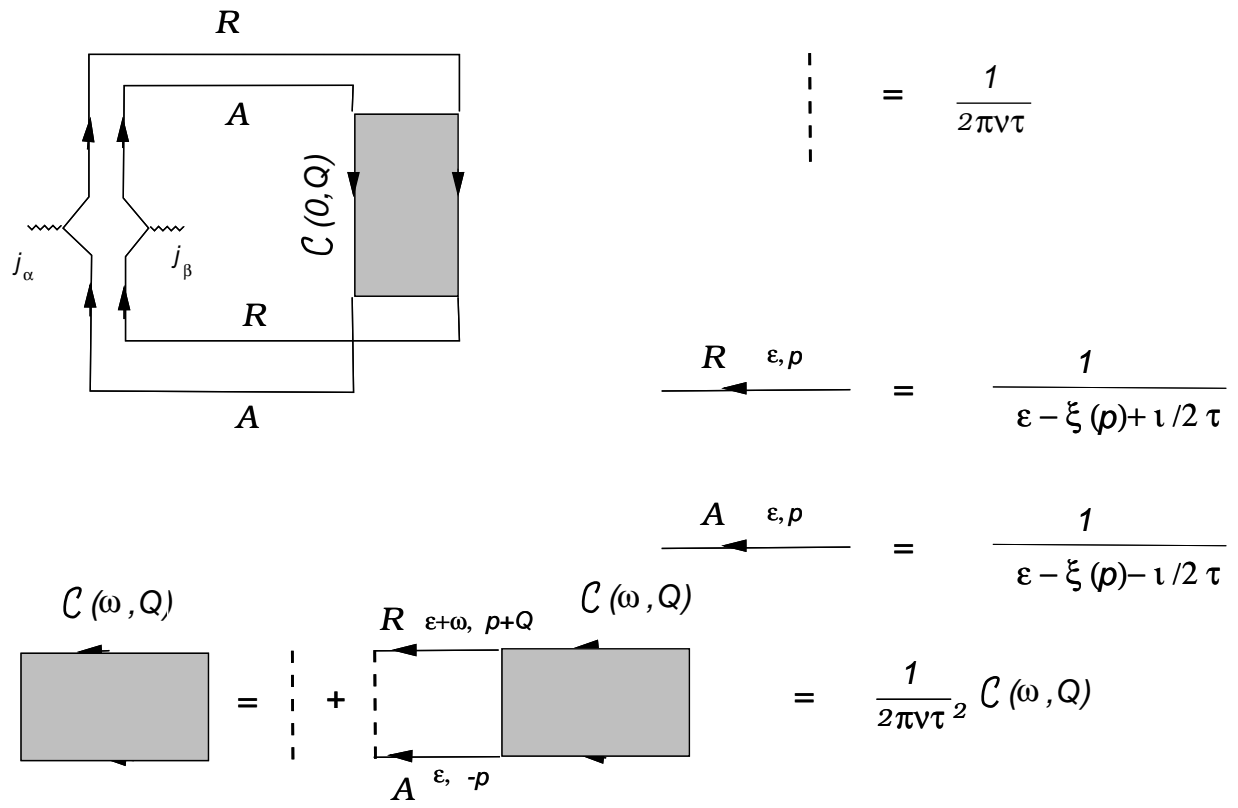
FIG. 5. Diagrams for the averaging of $\sigma^{n i}$. (a) Drude conductivity. (b) Weak localization correction 14 ; (c) More convenient for the further usage representation of diagram (b). Hereafter, solid lines denotes disorder average Green functions and dashed lines denote disorder.

Before averaging the interaction correction, it is convenient to Fourier transform Eqs. (5.13b) and (5.13c) over the difference $\boldsymbol{r}_{3}-\boldsymbol{r}_{4}$. We obtain from Eq. (5.13b)

$$
\begin{aligned}
& \sigma_{\alpha \beta}^{\mathrm{deph}}=-\frac{1}{4 \pi} \int \frac{d \omega}{2 \pi} \int \frac{d^{d} q}{(2 \pi)^{d}}\left(\frac{\omega}{2 T \sinh ^{2} \frac{\omega}{2 T}}\right)\left[\operatorname{Im}^{A} \mathcal{L}^{A}(\omega, q)\right]\left\{\left\langle\left\langle\hat{j}_{\alpha} G_{12}^{R}(\epsilon) \hat{j}_{\beta} G_{23}^{A}(\epsilon) G_{34}^{A}(\epsilon-\omega) G_{41}^{A}(\epsilon)\right\rangle\right\rangle_{34} \quad(5.1\right. \\
& \left.\quad+\left\langle\left\langle\hat{j}_{\alpha} G_{12}^{A}(\epsilon) \hat{j}_{\beta} G_{23}^{R}(\epsilon) G_{34}^{R}(\epsilon-\omega) G_{41}^{R}(\epsilon)\right\rangle\right\rangle_{34}+\left\langle\left\langle\hat{j}_{\alpha} G_{13}^{R}(\epsilon) G_{32}^{R}(\epsilon-\omega) \hat{j}_{\beta} G_{24}^{A}(\epsilon-\omega) G_{41}^{A}(\epsilon)\right\rangle\right\rangle_{34}+\alpha \leftrightarrow \beta\right\}
\end{aligned}
$$

where hereafter we use the short hand notation

$$
\left\langle\left\langle F_{12}^{(1)} F_{23}^{(2)} \ldots F_{n 1}^{(n)}\right\rangle\right\rangle_{i j} \equiv \int \frac{d \boldsymbol{r}_{1} \ldots d \boldsymbol{r}_{n}}{\mathcal{V}}\left\langle\left\langle F^{(1)}\left(\boldsymbol{r}_{1}, \boldsymbol{r}_{2}\right) F^{(2)}\left(\boldsymbol{r}_{2}, \boldsymbol{r}_{3}\right) \ldots F^{(n)}\left(\boldsymbol{r}_{n}, \boldsymbol{r}_{1}\right)\right\rangle\right\rangle \exp \left[i \boldsymbol{q}\left(\boldsymbol{r}_{i}-\boldsymbol{r}_{j}\right)\right]
$$

for any functions $F^{(i)}$.

Three last terms in brackets of Eq. (5.13d) can be simplified with the help of the identities

$$
\begin{array}{r}
\int d \boldsymbol{r}_{3} G^{R}\left(\epsilon, \boldsymbol{r}_{1}, \boldsymbol{r}_{3}\right) \hat{j}_{\alpha} G^{R}\left(\epsilon, \boldsymbol{r}_{3}, \boldsymbol{r}_{2}\right)=-i e\left(\boldsymbol{r}_{1}-\boldsymbol{r}_{2}\right)_{\alpha} G^{R}\left(\epsilon, \boldsymbol{r}_{1}, \boldsymbol{r}_{2}\right) \\
\int d \boldsymbol{r}_{3} d \boldsymbol{r}_{4} G^{R}\left(\epsilon, \boldsymbol{r}_{1}, \boldsymbol{r}_{3}\right) \hat{j}_{\alpha} G^{R}\left(\epsilon, \boldsymbol{r}_{3}, \boldsymbol{r}_{4}\right) \hat{j}_{\beta} G^{R}\left(\epsilon, \boldsymbol{r}_{4}, \boldsymbol{r}_{2}\right) \hat{j}_{\beta}= \\
-\frac{e^{2}}{2}\left(\boldsymbol{r}_{1}-\boldsymbol{r}_{2}\right)_{\alpha}\left(\boldsymbol{r}_{1}-\boldsymbol{r}_{2}\right)_{\beta} G^{R}\left(\epsilon, \boldsymbol{r}_{1}, \boldsymbol{r}_{2}\right)+\frac{e^{2} \delta_{\alpha \beta}}{2 m} \frac{\partial}{\partial \epsilon} G^{R}\left(\epsilon, \boldsymbol{r}_{1} \boldsymbol{r}_{2}\right),
\end{array}
$$

which follow from the gauge invariance of the theory and do not require any translational invariance, see Appendix. We find

$$
\begin{aligned}
& \sigma_{\alpha \beta}^{\mathrm{int}}=-\frac{1}{4 \pi} \operatorname{Im} \int \frac{d \omega}{2 \pi} \int \frac{d^{d} q}{(2 \pi)^{d}}\left[\frac{d}{d \omega}\left(\omega \operatorname{coth} \frac{\omega}{2 T}\right)\right] \mathcal{L}^{A}(\omega, q)\left\{\left\langle\left\langle\hat{j}_{\alpha} G_{12}^{A}(\epsilon) \hat{j}_{\beta} G_{23}^{R}(\epsilon) G_{34}^{R}(\epsilon-\omega) G_{41}^{R}(\epsilon)\right\rangle\right\rangle_{34}\right. \\
& +\left\langle\left\langle\hat{j}_{\alpha} G_{12}^{R}(\epsilon) \hat{j}_{\beta} G_{23}^{A}(\epsilon) G_{34}^{R}(\epsilon-\omega) G_{41}^{A}(\epsilon)\right\rangle\right\rangle_{34}+\frac{e^{2}}{2} \frac{\partial^{2}}{\partial q_{\alpha} \partial q_{\beta}}\left\langle\left\langle G_{12}^{A}(\epsilon) G_{21}^{R}(\epsilon-\omega)\right\rangle\right\rangle_{12} \\
& \left.\quad+2 e \frac{\partial}{\partial q_{\beta}}\left\langle\left\langle G_{31}^{R}(\epsilon) \hat{j}_{\alpha} G_{12}^{A}(\epsilon) G_{23}^{R}(\epsilon-\omega)\right\rangle\right\rangle_{23}+\alpha \leftrightarrow \beta\right\} .
\end{aligned}
$$

Deriving Eq. (5.16) we used the fact that

$$
\begin{aligned}
& \operatorname{Im} \int d \omega\left[\frac{d}{d \omega}\left(\omega \operatorname{coth} \frac{\omega}{2 T}\right)\right] \mathcal{L}^{A}(\omega, q)\left\langle\left\langle G_{21}^{R}(\epsilon-\omega) \partial_{\epsilon} G_{12}^{A}(\epsilon)\right\rangle\right\rangle_{12} \\
= & \int d \omega\left[\frac{d}{d \omega}\left(\omega \operatorname{coth} \frac{\omega}{2 T}\right)\right]\left[\mathcal{L}^{A}(\omega, q)\left\langle\left\langle G_{21}^{R}(\epsilon-\omega) \partial_{\epsilon} G_{12}^{A}(\epsilon)\right\rangle\right\rangle_{12}-\mathcal{L}^{R}(\omega, q)\left\langle\left\langle G_{12}^{A}(\epsilon-\omega) \partial_{\epsilon} G_{21}^{R}(\epsilon)\right\rangle\right\rangle_{12}\right] \\
= & \int d \omega\left[\frac{d}{d \omega}\left(\omega \operatorname{coth} \frac{\omega}{2 T}\right)\right] \mathcal{L}^{A}(\omega, q)\left[\left\langle\left\langle G_{21}^{R}(\epsilon-\omega) \partial_{\epsilon} G_{12}^{A}(\epsilon)\right\rangle\right\rangle_{12}+\left\langle\left\langle G_{12}^{A}(\epsilon+\omega) \partial_{\epsilon} G_{21}^{R}(\epsilon)\right\rangle\right\rangle_{12}\right] \\
= & \int d \omega\left[\frac{d}{d \omega}\left(\omega \operatorname{coth} \frac{\omega}{2 T}\right)\right] \mathcal{L}^{A}(\omega, q)\left[\partial_{\epsilon}\left\langle\left\langle G_{21}^{R}(\epsilon-\omega) G_{12}^{A}(\epsilon)\right\rangle\right\rangle_{12}+\left\langle\left\langle G_{12}^{A}(\epsilon+\omega) \partial_{\epsilon} G_{21}^{R}(\epsilon)\right\rangle\right\rangle_{12}-\left\langle\left\langle G_{12}^{A}(\epsilon) \partial_{\epsilon} G_{21}^{R}(\epsilon-\omega)\right\rangle\right\rangle_{12}\right] \\
= & 0 .
\end{aligned}
$$

Now we are in the position to perform actual disorder average of the products entering into Eqs. (5.14) and (5.16). Diagrams for all the terms from Eq. (5.14) are shown in Fig. 6. They can be obtained by cutting the corresponding Green functions on the diagrams of Fig. 5b. The analytic expression for these diagrams is

$$
\begin{aligned}
\left\langle\left\langle\hat{j}_{\alpha} G_{12}^{R}(\epsilon) \hat{j}_{\beta} G_{23}^{A}(\epsilon) G_{34}^{A}(\epsilon-\omega) G_{41}^{A}(\epsilon)\right\rangle\right\rangle_{34} & =\left\langle\left\langle\hat{j}_{\alpha} G_{12}^{A}(\epsilon) \hat{j}_{\beta} G_{23}^{R}(\epsilon) G_{34}^{R}(\epsilon-\omega) G_{41}^{R}(\epsilon)\right\rangle\right\rangle_{34} \\
& =\delta_{\alpha \beta} \frac{2 \sigma}{\nu} \int \frac{d^{d} Q}{(2 \pi)^{d}} \mathcal{C}^{2}(0, \boldsymbol{Q}) \mathcal{C}(-\omega, \boldsymbol{Q}+\boldsymbol{q}) \\
\left\langle\left\langle\hat{j}_{\alpha} G_{13}^{R}(\epsilon) G_{32}^{R}(\epsilon-\omega) \hat{j}_{\beta} G_{24}^{A}(\epsilon-\omega) G_{41}^{A}(\epsilon)\right\rangle\right\rangle_{34} & =-\delta_{\alpha \beta} \frac{4 \sigma}{\nu} \int \frac{d^{d} Q}{(2 \pi)^{d}}|\mathcal{C}(\omega, \boldsymbol{Q})|^{2} \mathcal{C}(0, \boldsymbol{Q}+\boldsymbol{q})
\end{aligned}
$$


where the Cooperon propagator is defined by Eq. (2.45). Substitution of Eqs. (5.17) and (5.8) into Eq. (5.14) immediately yields Eq. (4.5).
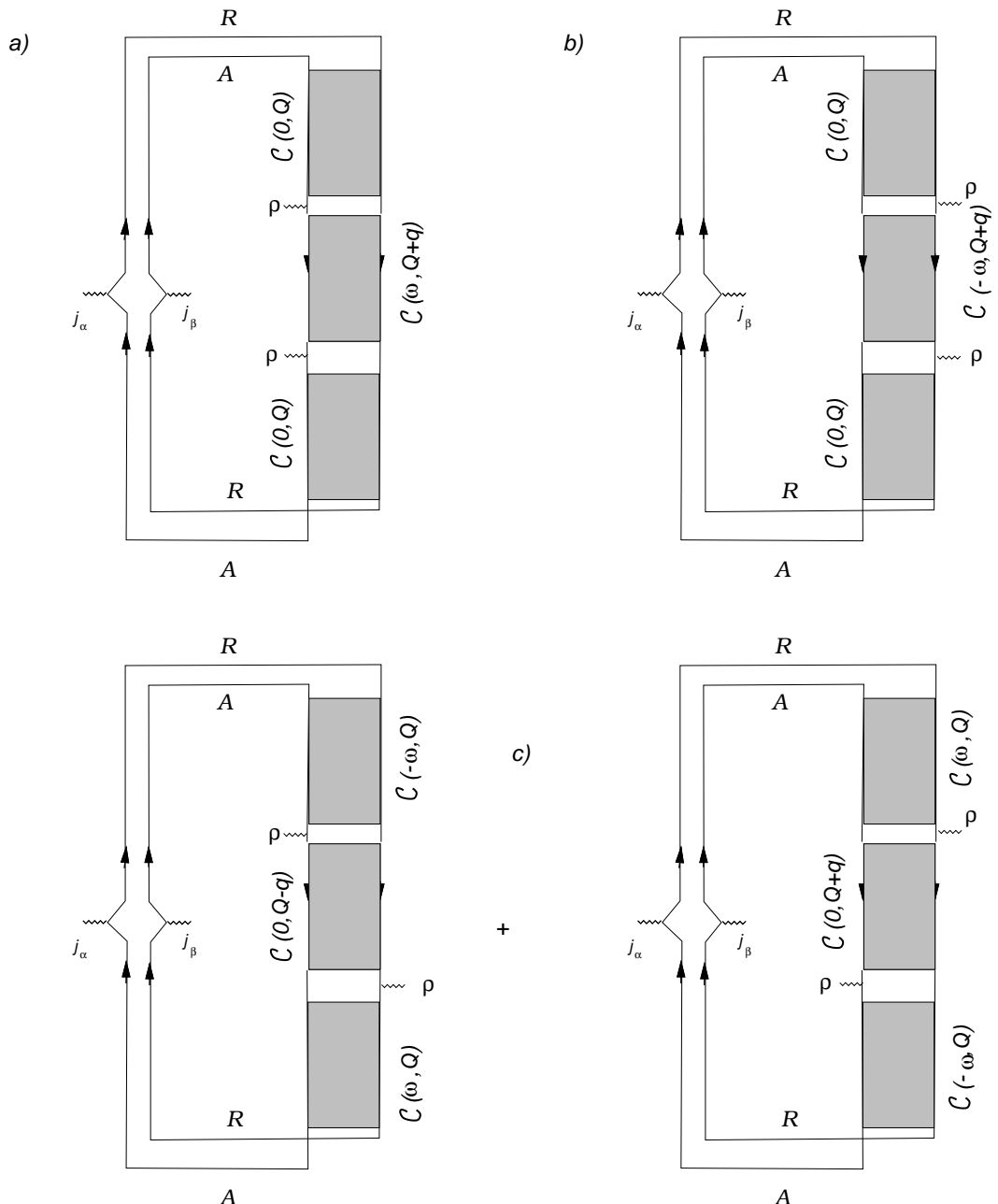

A

FIG. 6. Diagrams for the averaging of products (a) $\left\langle\left\langle\hat{j}_{\alpha} G_{12}^{R} \hat{j}_{\beta} G_{23}^{A} G_{34}^{A} G_{41}^{A}\right\rangle\right\rangle_{34}$; (b) $\left\langle\left\langle\hat{j}_{\alpha} G_{12}^{A} \hat{j}_{\beta} G_{23}^{R} G_{34}^{R} G_{41}^{R}\right\rangle\right\rangle_{34}$; and (c) $\left\langle\left\langle\hat{j}_{\alpha} G_{13}^{R} G_{32}^{R} \hat{j}_{\beta} G_{24}^{A} G_{41}^{A}\right\rangle\right\rangle_{34}$

Diagrams for the averaging of the terms entering into Eq. (5.16) are a little bit more complicated, see Figs. 7 - 9 . The analytic expression for the diagrams in Fig. 7 is

$$
\begin{gathered}
\left\langle\left\langle\hat{j}_{\alpha} G_{12}^{R}(\epsilon) \hat{j}_{\beta} G_{23}^{A}(\epsilon) G_{34}^{R}(\epsilon-\omega) G_{41}^{A}(\epsilon)\right\rangle\right\rangle_{34}=2 \pi i \sigma \delta_{\alpha \beta} \frac{\partial}{\partial \omega} \mathcal{D}(-\omega, q)+i \frac{2 \sigma}{\nu} \delta_{\alpha \beta} \frac{\partial}{\partial \omega}\left[D q^{2} \mathcal{D}^{2}(-\omega, q) \int \frac{d^{d} Q}{(2 \pi)^{d}} \mathcal{C}(-\omega, Q)\right] \\
-\delta_{\alpha \beta} \frac{2 \sigma}{\nu} \int \frac{d^{d} Q}{(2 \pi)^{d}}\left[\mathcal{C}^{2}(0, \boldsymbol{Q}) \mathcal{D}(-\omega, \boldsymbol{q})+\mathcal{C}(0, \boldsymbol{Q}) \mathcal{D}^{2}(-\omega, \boldsymbol{q})\right] \\
+\frac{4 \sigma}{\nu} \mathcal{D}^{2}(-\omega, \boldsymbol{q}) \int \frac{d^{d} Q}{(2 \pi)^{d}} \mathcal{C}(-\omega, \boldsymbol{Q}) \mathcal{C}(0, \boldsymbol{Q}-\boldsymbol{q})\left(D q_{\alpha} Q_{\beta}+D Q_{\alpha} q_{\beta}\right),
\end{gathered}
$$

where Cooperon propagator $\mathcal{C}(-\omega, \boldsymbol{Q})$ is defined by Eq. (2.45) and the diffuson is given by

$$
\mathcal{D}(\omega, Q)=\frac{1}{-i \omega+D Q^{2}} .
$$

The first term in Eq. (5.18) is the leading classical approximation, Fig. Fa and all the other terms come from the interference corrections which are shown term by term in Fig. $7 \mathrm{~b}$-d. 
a)

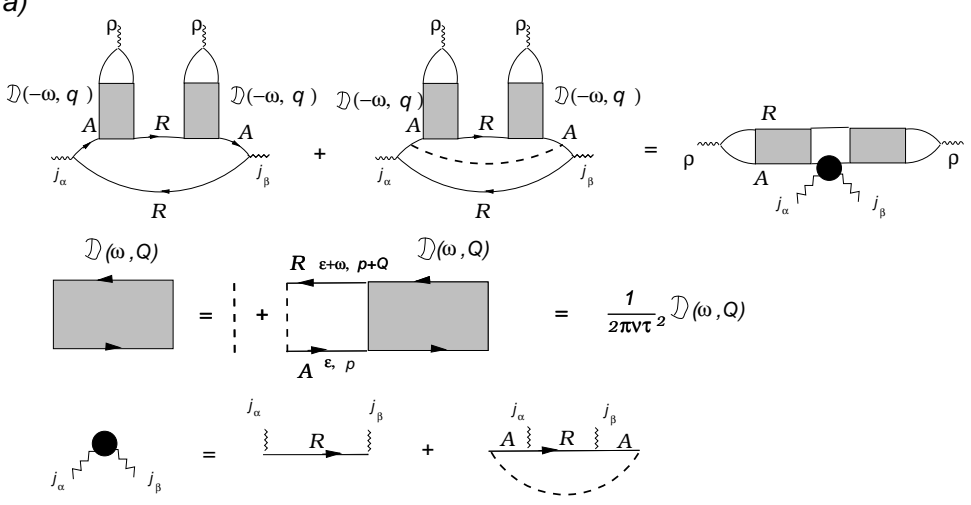

b)
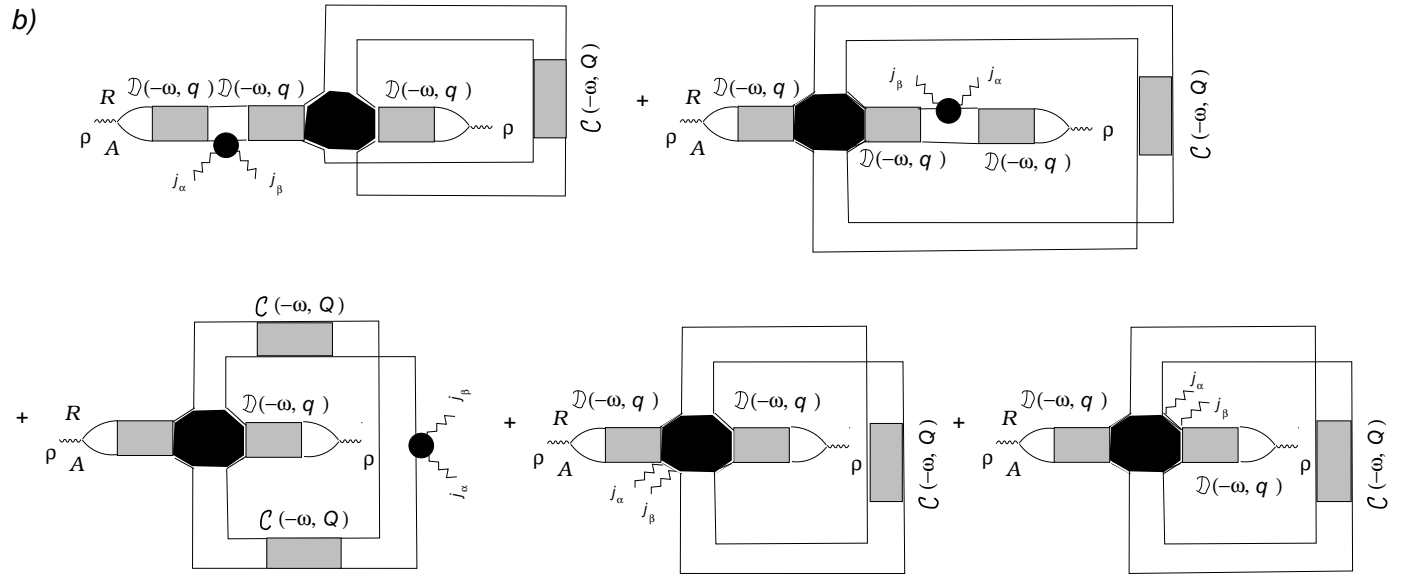

c)
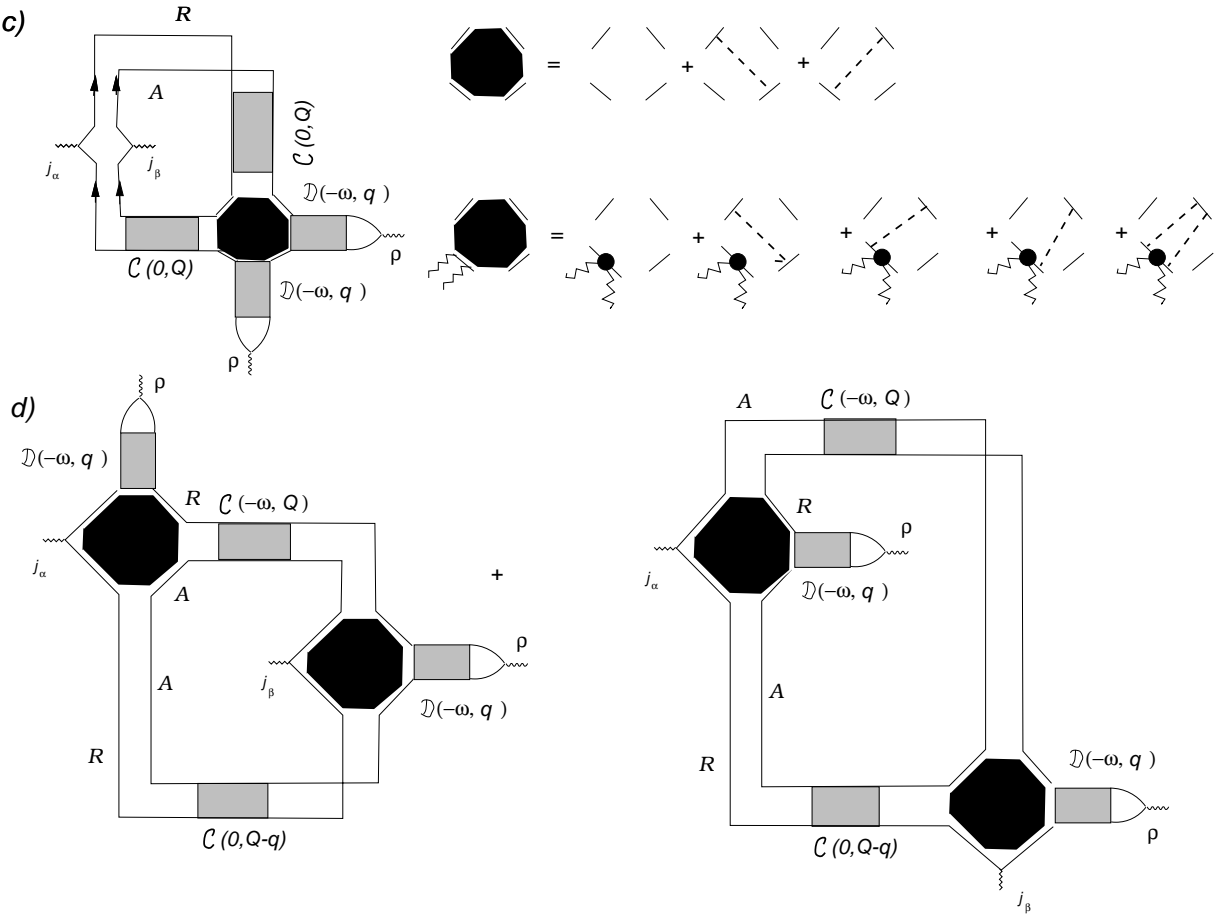

FIG. 7. Diagrams for the averaging of products $\left\langle\left\langle\hat{j}_{\alpha} G_{12}^{R} \hat{j}_{\beta} G_{23}^{A} G_{34}^{R} G_{41}^{A}\right\rangle\right\rangle_{34}$.

The third term in brackets in Eq. (5.16) is the density - density correlation function with the well known answer, 
see Fig. (8)

$$
\left\langle\left\langle G_{12}^{A}(\epsilon) G_{21}^{R}(\epsilon-\omega)\right\rangle\right\rangle_{12}=4 \pi \nu \mathcal{D}(-\omega, q)+4 D q^{2} \mathcal{D}^{2}(-\omega, q) \int \frac{d^{d} Q}{(2 \pi)^{d}} \mathcal{C}(-\omega, Q),
$$

where the last term is nothing but the weak-localization correction to the diffusion constant.

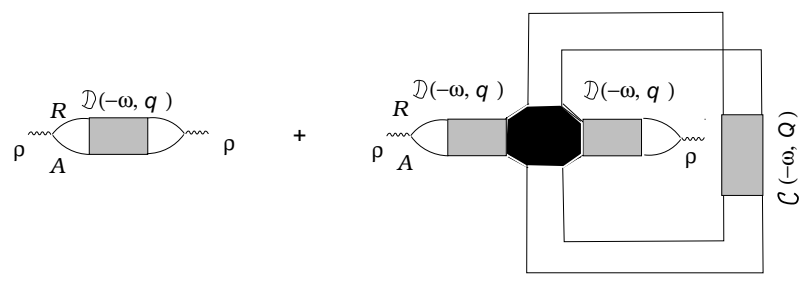

FIG. 8. Diagrams for the averaging of density-density correlation function $\left\langle\left\langle G_{12}^{R} G_{21}^{A}\right\rangle\right\rangle_{12}$.

Finally, the last term in brackets in Eq. (5.16) is averaged according to Fig. 9. The analytic expression for this diagram is

$$
\left\langle\left\langle G_{31}^{R}(\epsilon) \hat{j}_{\alpha} G_{12}^{A}(\epsilon) G_{23}^{R}(\epsilon-\omega)\right\rangle\right\rangle_{23}=4 e D q_{\alpha} \mathcal{D}(-\omega, q) \int \frac{d^{d} Q}{(2 \pi)^{d}} \mathcal{C}(0, Q) \mathcal{C}(-\omega, \boldsymbol{Q}+\boldsymbol{q}) .
$$

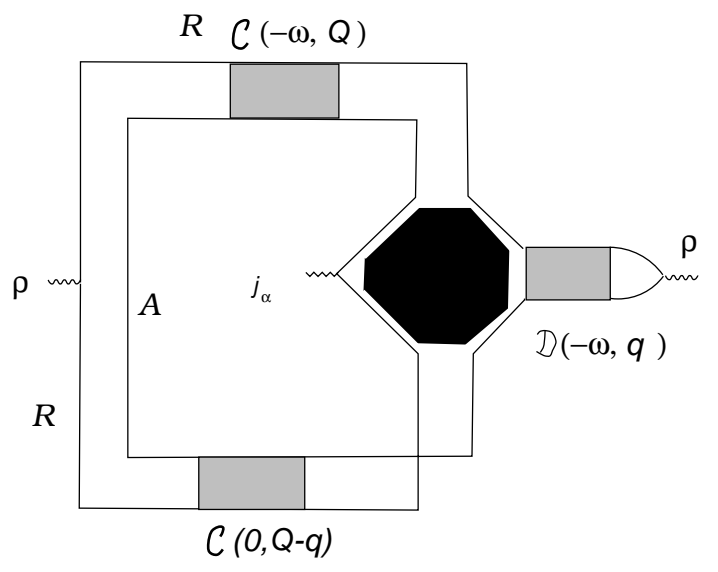

FIG. 9. Diagrams for the averaging of product $\left\langle\left\langle G_{31}^{R} \hat{j}_{\alpha} G_{12}^{A} G_{23}^{R}\right\rangle\right\rangle_{23}$.

Now, we are ready to calculate the interaction correction (5.16). First we take into account leading $1 / \mathrm{g}$ approximation only, i.e. retain the first terms in Eq. (5.18) and (5.20), neglect terms (5.17a $)$ and (5.21), and use the interaction propagator $\mathcal{L}^{A}$ from (5.8) with the bare diffusion constant $D$. It immediately gives the known result, Eq. (2.27).

In order to find the cross-term of weak interaction and weak localization, we have to take into account not only all the remaining terms in Eqs. (5.17a), (5.18), 5.20) and (5.21) but also the weak localization correction to the interaction propagator (5.8) in the leading approximation

$$
\mathcal{L}^{A}(\omega, q) \approx-\frac{1}{2 \nu}\left[\frac{i \omega+D q^{2}}{D q^{2}}\right]-\frac{i \omega}{2 \nu D q^{2}}\left(\frac{1}{\pi \nu}\right) \int \frac{d^{d} Q}{(2 \pi)^{d}} \mathcal{C}(-\omega, \boldsymbol{Q}),
$$

where the last term is the weak localization correction to the diffusion constant.

Substituting Eqs. (5.17a), (5.18) and (5.20) - 5.22) into Eq. (5.16) and keeping only terms of the second order in $1 / g$, i.e. only those involving two integrations over the wavevectors $Q, q$, we obtain 


$$
\begin{aligned}
\sigma_{\alpha \beta}^{\mathrm{CWL}}= & \frac{\sigma}{2 \pi \nu^{2}} \operatorname{Im} \int \frac{d \omega}{2 \pi}\left[\frac{d}{d \omega}\left(\omega \operatorname{coth} \frac{\omega}{2 T}\right)\right] \int \frac{d^{d} q d^{d} \boldsymbol{Q}}{(2 \pi)^{2 d}} \\
& \times\left\{\frac{\delta_{\alpha \beta}}{D q^{2}}\left[\mathcal{C}^{2}(0, \boldsymbol{Q})\left(\frac{\mathcal{C}(-\omega, \boldsymbol{Q}+\boldsymbol{q})}{\mathcal{D}(-\omega, \boldsymbol{q})}-1\right)-\mathcal{C}(0, \boldsymbol{Q}) \mathcal{D}(-\omega, \boldsymbol{q})+\mathcal{C}(-\omega, \boldsymbol{Q}) \mathcal{D}(-\omega, \boldsymbol{q})+2 \mathcal{C}(-\omega, \boldsymbol{Q}+\boldsymbol{q}) \mathcal{C}(0, \boldsymbol{Q})\right]\right. \\
& +\frac{2 q_{\alpha}}{D q^{2}} \frac{\partial}{\partial q_{\beta}} \mathcal{C}(-\omega, \boldsymbol{Q}+\boldsymbol{q}) \mathcal{C}(0, \boldsymbol{Q})-4 \frac{q_{\alpha} q_{\beta}}{q^{2}} \mathcal{D}(-\omega, \boldsymbol{q}) \mathcal{C}(-\omega, \boldsymbol{Q}+\boldsymbol{q}) \mathcal{C}(0, \boldsymbol{Q}) \\
& \left.+2 \frac{q_{\alpha} Q_{\beta}+Q_{\alpha} q_{\beta}}{q^{2}} \mathcal{D}(-\omega, \boldsymbol{q}) \mathcal{C}(0, \boldsymbol{Q}-\boldsymbol{q}) \mathcal{C}(-\omega, \boldsymbol{Q})+\delta_{\alpha \beta} \mathcal{D}(-\omega, \boldsymbol{q}) \mathcal{C}^{2}(-\omega, \boldsymbol{Q})+8 D q_{\alpha} q_{\beta} \mathcal{D}^{3}(-\omega, \boldsymbol{q}) \mathcal{C}(-\omega, \boldsymbol{Q})\right\}
\end{aligned}
$$

Derivation of Eq. (4.7) from Eq. (5.23) is now just a matter of a lengthy albeit straightforward calculation. We will write most important intermediate formulas below. Integration over the wavevectors is performed first. To carry out this integration, it is convenient to represent each Cooperon and diffuson propagator by additional time integral

$$
\mathcal{C}(\omega, \boldsymbol{Q})=\int_{0}^{\infty} d t \exp \left(-\frac{t}{\mathcal{C}(\omega, \boldsymbol{Q})}\right)
$$

After this manipulation, the integrals over wavevectors become Gaussian and can be immediately calculated. We find

$$
\begin{aligned}
I_{1} & \equiv \int \frac{d^{d} q d^{d} Q}{(2 \pi)^{2 d}} \frac{1}{D q^{2}}\left\{\mathcal{C}^{2}(0, \boldsymbol{Q})\left[\frac{\mathcal{C}(-\omega, \boldsymbol{Q}+\boldsymbol{q})}{\mathcal{D}(-\omega, \boldsymbol{q})}-1\right]+[\mathcal{C}(-\omega, \boldsymbol{Q})-\mathcal{C}(0, \boldsymbol{Q})] \mathcal{D}(-\omega, \boldsymbol{q})+2 \mathcal{C}(-\omega, \boldsymbol{Q}+\boldsymbol{q}) \mathcal{C}(0, \boldsymbol{Q})\right\} \\
& =\frac{\Gamma^{2}\left(2-\frac{d}{2}\right)}{(4 \pi D)^{d}}\left\{\frac{4}{2-d}(i \omega)^{d / 2-2}\left(\frac{1}{\tau_{H}}\right)^{d / 2-1}-\left[\frac{2}{2-d}\right]^{2}\left[1+(1-d) \frac{\Gamma(3-d) \Gamma(d / 2)}{\Gamma(2-d / 2)}\right](i \omega)^{d-3}\right\},
\end{aligned}
$$

where $\Gamma(x)$ is the Gamma function. Equation (5.25a is written with an accuracy up to term of the order of $\omega^{d-3}\left(\omega \tau_{H}\right)^{-d / 2}$. This accuracy however is sufficient for our purposes, since all the Cooperons and diffusons here are functions analytic in the complex semiplane $\operatorname{Im} \omega<0$, and therefore integration over $\omega$ is determined by $\omega \gtrsim T$. On the other hand, we are considering the case $T \tau_{H} \gg 1$, and all the corrections to Eq. (5.25) are smaller by this parameter. Other integrals calculated in the same approximation are given by

$$
\begin{aligned}
& I_{2} \equiv \int \frac{d^{d} q d^{d} Q}{(2 \pi)^{2 d}} \frac{q_{\alpha}}{D q^{2}} \frac{\partial}{\partial q_{\beta}} \mathcal{C}(-\omega, \boldsymbol{Q}+\boldsymbol{q}) \mathcal{C}(0, \boldsymbol{Q})= \\
&=-\frac{2 \delta_{\alpha \beta}}{d} \frac{\Gamma^{2}\left(2-\frac{d}{2}\right)}{(4 \pi D)^{d}}\left\{\frac{2}{2-d}(i \omega)^{d / 2-2}\left(\frac{1}{\tau_{H}}\right)^{d / 2-1}-\frac{2}{2-d} \frac{\Gamma(3-d) \Gamma(d / 2)}{\Gamma(2-d / 2)}(i \omega)^{d-3}\right\} \\
& I_{3} \equiv \int \frac{d^{d} q d^{d} Q}{(2 \pi)^{2 d}} \frac{q_{\alpha} q_{\beta}}{q^{2}} \mathcal{D}(-\omega, \boldsymbol{q}) \mathcal{C}(-\omega, \boldsymbol{Q}+\boldsymbol{q}) \mathcal{C}(0, \boldsymbol{Q}) \\
&=\frac{\delta_{\alpha \beta}}{d} \frac{2}{2-d} \frac{\Gamma^{2}\left(2-\frac{d}{2}\right)}{(4 \pi D)^{d}}\left[(i \omega)^{d / 2-2}\left(\frac{1}{\tau_{H}}\right)^{d / 2-1}-\frac{1}{3-d}(i \omega)^{d-3}\right] \\
& I_{4} \equiv \int \frac{d^{d} q d^{d} Q}{(2 \pi)^{2 d}} \frac{q_{\alpha} Q_{\beta}}{q^{2}} \mathcal{D}(-\omega, \boldsymbol{q}) \mathcal{C}(0, \boldsymbol{Q}-\boldsymbol{q}) \mathcal{C}(-\omega, \boldsymbol{Q}) \\
&= \frac{\delta_{\alpha \beta}}{d} \frac{2}{2-d} \frac{\Gamma^{2}\left(2-\frac{d}{2}\right)}{(4 \pi D)^{d}}\left[(i \omega)^{d / 2-2}\left(\frac{1}{\tau_{H}}\right)^{d / 2-1}-\frac{1}{3-d}(i \omega)^{d-3}\right] \\
&-\frac{\delta_{\alpha \beta}}{d} \frac{(i \omega)^{d-3}}{(4 \pi D)^{d}} \int_{0}^{\infty} \frac{d x d y d z}{(x y+x z+y z)^{d / 2}}\left(\frac{y}{y+z}\right) e^{-x-y} ; \\
& I_{5} \equiv \int \frac{d^{d} q d^{d} Q}{(2 \pi)^{2 d}} \mathcal{D}(-\omega, \boldsymbol{q}) \mathcal{C}^{2}(-\omega, \boldsymbol{Q})=\frac{2}{2-d} \frac{\Gamma^{2}\left(2-\frac{d}{2}\right)}{(4 \pi D)^{d}}(i \omega)^{d-3}
\end{aligned}
$$




$$
I_{6} \equiv \int \frac{d^{d} q d^{d} Q}{(2 \pi)^{2 d}} D q_{\alpha} q_{\beta} \mathcal{D}^{3}(-\omega, \boldsymbol{q}) \mathcal{C}(-\omega, \boldsymbol{Q})=\frac{\delta_{\alpha \beta}}{4(2-d)} \frac{\Gamma^{2}\left(2-\frac{d}{2}\right)}{(4 \pi D)^{d}}(i \omega)^{d-3} .
$$

Substituting Eqs. (5.25) into Eq. (5.23), retaining only most singular at $d \rightarrow 2$ coefficients in front of $\omega^{d-3}$, and omitting indices $\alpha, \beta$, we find

$$
\delta \sigma_{\mathrm{CWL}}=\frac{\sigma}{2 \pi \nu^{2}} \frac{1}{(4 \pi D)^{d}} \operatorname{Im} \int \frac{d \omega}{2 \pi}\left[\frac{d}{d \omega}\left(\omega \operatorname{coth} \frac{\omega}{2 T}\right)\right]\left[-\frac{4 \Gamma^{2}(2-d / 2)}{d}(i \omega)^{d / 2-2}\left(\frac{1}{\tau_{H}}\right)^{d / 2-1}+\frac{4}{2-d}(i \omega)^{d-3}\right] .
$$

Using formula

$$
\operatorname{Im} \int_{-\infty}^{\infty} \frac{d x}{(i x)^{\alpha}} \frac{d}{d x}\left(x \operatorname{coth} \frac{x}{2}\right)=-\frac{2 \alpha}{(2 \pi)^{\alpha-1}} \zeta(\alpha)
$$

with $\zeta(x)$ being Riemann zeta-function, we obtain Eq. (4.7).

\section{DISCUSSION OF THE RESULTS}

\section{A. Theory}

In Sec. \, we demonstrated explicitly that dephasing can be caused only by e-e collisions with the energy transfer smaller or of the order of temperature, see Eq. (4.5). This conclusion clearly contradicts the results of Ref. 9. It means that the procedure proposed in Ref. 9 fails already on the level of the first order perturbation theory in the e-e interactions. This calculation is sufficient to make the conclusion that the "old" rather than "new" theory describes the temperature dependence of the dephasing rate correctly and thus to end the pointless discussion. However, the errors in Ref. 9 originate from a quite typical misuse of the semiclassical approximations. For this reason we would like to highlight these errors and to discuss them in more detail.

According to Eqs. (2.45), (4.5) and (3.3), dephasing can be found by determining the coefficient in front of the term $[\mathcal{C}(\boldsymbol{Q}, 0)]^{2}$. The $Q \rightarrow O$ and $\tau \rightarrow \infty$ limit of this coefficient has a meaning of the inverse dephasing time $1 / \tau_{\phi}$, while its $Q$ and $H$-dependences represent renormalization of the diffusion constant $D$ and of the residue of the Cooper pole respectively. These renormalizations contribute eventually to the interaction correction to the conductivity (4.7).

Therefore we can focus only on the first term in brackets in Eq. (5.23):

$$
\sigma^{?}=-\frac{\sigma}{2 \pi \nu^{2}} \operatorname{Im} \int \frac{d \omega}{2 \pi}\left[\frac{d}{d \omega}\left(\omega \operatorname{coth} \frac{\omega}{2 T}\right)\right] \int \frac{d^{d} q d^{d} Q}{(2 \pi)^{2 d}} \mathcal{L}^{A}(\omega, q) \mathcal{C}^{2}(0, \boldsymbol{Q})[\mathcal{C}(-\omega, \boldsymbol{Q}+\boldsymbol{q})-\mathcal{D}(-\omega, \boldsymbol{q})]
$$

Note that in the corresponding equation of Ref. 9, see Eq. (3.3), only the first Cooperon term in the brackets is present. The second diffuson term changes the result dramatically since at $Q \rightarrow O$ and $\tau_{H} \rightarrow \infty$ it simply cancels the first one.

In order to understand why the diffuson term and thus the cancellation are missed in Ref. 9, let us examine step by step the derivation proposed in that paper.

1. Authors of Ref. 9 used Keldysh technique and decoupled the interaction by Hubbard-Stratonovich transformation. This step is rather standard and the result in the perturbation theory should be consistent with our Eq. 5.12.

2. Their next step was to write down the formally exact path integral expression for Green functions in a disordered potential

$$
\begin{aligned}
& G^{R}\left(\epsilon ; \boldsymbol{r}_{1}, \boldsymbol{r}_{2}\right)=\int_{0}^{\infty} d t e^{i \epsilon t} \int_{\boldsymbol{r}(0)=\boldsymbol{r}_{1}}^{\boldsymbol{r}(t)=\boldsymbol{r}_{2}} \mathcal{D}[\boldsymbol{r}(t)] \exp \left(\frac{i}{\hbar} \mathcal{S}[\boldsymbol{r}(t)]\right) \\
& G^{A}\left(\epsilon ; \boldsymbol{r}_{1}, \boldsymbol{r}_{2}\right)=\int_{-\infty}^{0} d t e^{i \epsilon t} \int_{\boldsymbol{r}(0)=\boldsymbol{r}_{1}}^{\boldsymbol{r}(t)=\boldsymbol{r}_{2}} \mathcal{D}[\boldsymbol{r}(t)] \exp \left(-\frac{i}{\hbar} \mathcal{S}[\boldsymbol{r}(t)]\right)
\end{aligned}
$$

where $\mathcal{S}[\boldsymbol{r}(t)]$ is the classical action over the trajectory $\boldsymbol{r}(t)$. 
3. Afterwards an averaging of products of Green functions was performed. During this average only some paths were selected by hand. It is this selection that, as far as we understand, causes all the errors.

Indeed, consider the average

$$
\left\langle\left\langle G^{R}\left(\epsilon ; \boldsymbol{r}_{1}, \boldsymbol{r}_{2}\right) G^{A}\left(\epsilon ; \boldsymbol{r}_{1}, \boldsymbol{r}_{2}\right)\right\rangle\right\rangle=2 \pi \nu \mathcal{D}\left(0 ; \boldsymbol{r}_{1}, \boldsymbol{r}_{2}\right), \quad \mathcal{D}\left(\omega ; \boldsymbol{r}_{1}, \boldsymbol{r}_{2}\right)=\int \frac{d^{d} Q}{(2 \pi)^{d}} \frac{e^{i \boldsymbol{Q}\left(\boldsymbol{r}_{1}-\boldsymbol{r}_{2}\right)}}{-i \omega+D Q^{2}}
$$

Here, for simplicity, we consider the case of zero magnetic field and do not distinguish between diffusons and Cooperons. This average can be obtained semiclassically by pairing the paths shown in Fig. 10a and it is reproduced correctly in Ref. 9. The same is true for the simple average involving four coordinates, see Fig. 10b:

$$
\left\langle\left\langle G^{A}\left(\epsilon ; \boldsymbol{r}_{1}, \boldsymbol{r}_{2}\right) G^{R}\left(\epsilon ; \boldsymbol{r}_{1}, \boldsymbol{r}_{3}\right) G^{R}\left(\epsilon-\omega ; \boldsymbol{r}_{3}, \boldsymbol{r}_{4}\right) G^{R}\left(\epsilon ; \boldsymbol{r}_{4}, \boldsymbol{r}_{2}\right)\right\rangle\right\rangle=-2 \pi \nu \mathcal{D}\left(0 ; \boldsymbol{r}_{1}, \boldsymbol{r}_{3}\right) \mathcal{D}\left(-\omega ; \boldsymbol{r}_{3}, \boldsymbol{r}_{4}\right) \mathcal{D}\left(0 ; \boldsymbol{r}_{4}, \boldsymbol{r}_{2}\right)
$$

This expression corresponds to the diagram (6)a.

However, the other higher order products involve intersection of the classical paths, see Fig. 10c. Such configurations contribute in, e.g., average of the type

$$
\begin{aligned}
\left\langle\left\langle G^{R}\left(\epsilon ; \boldsymbol{r}_{1}, \boldsymbol{r}_{2}\right)\right.\right. & \left.\left.G^{A}\left(\epsilon ; \boldsymbol{r}_{1}, \boldsymbol{r}_{3}\right) G^{R}\left(\epsilon-\omega ; \boldsymbol{r}_{3}, \boldsymbol{r}_{4}\right) G^{A}\left(\epsilon ; \boldsymbol{r}_{4}, \boldsymbol{r}_{2}\right)\right\rangle\right\rangle \\
& =2 \pi \nu \int d \boldsymbol{r}_{5}\left\{i \omega \mathcal{D}\left(0 ; \boldsymbol{r}_{1}, \boldsymbol{r}_{5}\right) \mathcal{D}\left(-\omega ; \boldsymbol{r}_{5}, \boldsymbol{r}_{3}\right) \mathcal{D}\left(-\omega ; \boldsymbol{r}_{5}, \boldsymbol{r}_{4}\right) \mathcal{D}\left(0 ; \boldsymbol{r}_{2}, \boldsymbol{r}_{5}\right)\right. \\
& +D\left[\boldsymbol{\nabla}_{5} \mathcal{D}\left(0 ; \boldsymbol{r}_{1}, \boldsymbol{r}_{5}\right) \mathcal{D}\left(-\omega ; \boldsymbol{r}_{5}, \boldsymbol{r}_{4}\right)\right]\left[\boldsymbol{\nabla}_{5} \mathcal{D}\left(-\omega ; \boldsymbol{r}_{5}, \boldsymbol{r}_{3}\right) \mathcal{D}\left(0 ; \boldsymbol{r}_{2}, \boldsymbol{r}_{5}\right)\right] \\
& +2 D\left[\boldsymbol{\nabla}_{5} \mathcal{D}\left(0 ; \boldsymbol{r}_{1}, \boldsymbol{r}_{5}\right)\right]\left[\boldsymbol{\nabla}_{5} \mathcal{D}\left(-\omega ; \boldsymbol{r}_{5}, \boldsymbol{r}_{4}\right)\right] \mathcal{D}\left(-\omega ; \boldsymbol{r}_{5}, \boldsymbol{r}_{3}\right) \mathcal{D}\left(0 ; \boldsymbol{r}_{2}, \boldsymbol{r}_{5}\right) \\
& \left.+2 D \mathcal{D}\left(0 ; \boldsymbol{r}_{1}, \boldsymbol{r}_{5}\right) \mathcal{D}\left(-\omega ; \boldsymbol{r}_{5}, \boldsymbol{r}_{4}\right)\left[\boldsymbol{\nabla}_{5} \mathcal{D}\left(-\omega ; \boldsymbol{r}_{5}, \boldsymbol{r}_{3}\right)\right]\left[\boldsymbol{\nabla}_{5} \mathcal{D}\left(0 ; \boldsymbol{r}_{2}, \boldsymbol{r}_{5}\right)\right]\right\}
\end{aligned}
$$

see diagram in Fig. 7c. The point $\boldsymbol{r}_{5}$ has the meaning of the intersection point between classical trajectories. These intersections were not taken into account at all in Ref. 9 .

After performing the Fourier transforms over the coordinate differences $\boldsymbol{r}_{3}-\boldsymbol{r}_{4}$ and $\boldsymbol{r}_{1}-\boldsymbol{r}_{2}$, one finds

$$
\begin{aligned}
& \text { 6.4 } \rightarrow-\frac{2 \pi \nu}{\left[D Q^{2}\right]^{2}\left[i \omega+D(\boldsymbol{Q}-\boldsymbol{q})^{2}\right]} \\
& 6.5) \rightarrow \frac{2 \pi \nu}{\left[D Q^{2}\right]^{2}\left[i \omega+D q^{2}\right]}+\frac{2 \pi \nu}{\left[D Q^{2}\right]\left[i \omega+D q^{2}\right]^{2}}-\frac{4 \pi \nu D \boldsymbol{Q} \boldsymbol{q}}{\left[D Q^{2}\right]^{2}\left[i \omega+D q^{2}\right]^{2}}
\end{aligned}
$$

where the last term will vanish after the angular integration. One can see that signs of Eqs. (6.3) and (6.4) are opposite, which eventually leads to the cancellation in Eq. (6.1). 
a)

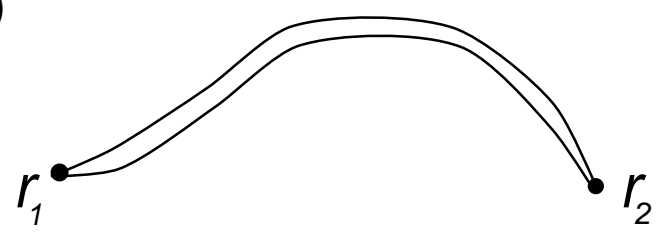

b)

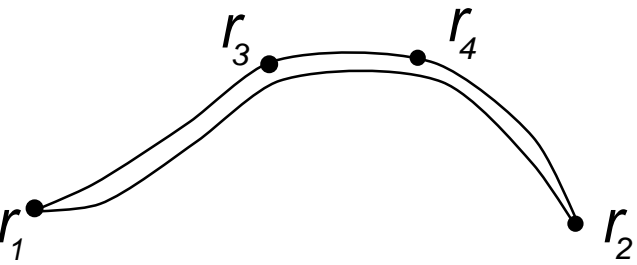

c)

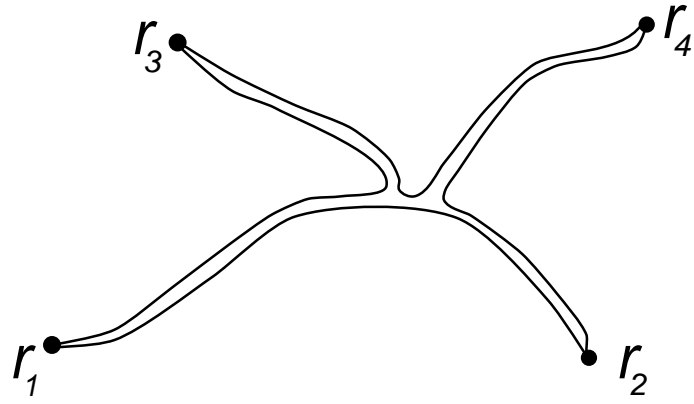

FIG. 10. Example of the classical paths contributing to the averages of products of the Green functions.

In fact, taking into account the switches between intersection of the classical paths is very crucial: mistreatment of such switches leads, e.g. to the violation of the conservation of number of particles and all the theoretical predictions of similar quality! In the diagrammatic technique such switches are described by so-called Hikami boxes (the shaded boxes in Fig. 7 - 9). Semiclassical approach usually has difficulties in the description of the transitions between the classical trajectories. Physical reasons for this difficulty were first discussed by Larkin and Ovchinnikov 35 in application to the superconductivity, and in Ref. 36 in application to the weak localization.

\section{B. Experiment}

Let us compare the available experimental results with predictions of the theory by Golubev and Zaikin $\mathrm{g}$. Authors concluded that "according to Eqs. (81), [of Ref. 9] in 2d and 3d systems the decoherence time becomes independent on $T$ already at relatively high temperatures. In the $3 \mathrm{~d}$ case such temperatures can be of the order of the inverse transport time, while for $2 \mathrm{~d}$ systems we have $T_{q} \sim\left(2 \tau \ln \left(p_{F}^{2} a l\right)\right)^{-1}$ ". If we now recall that the momentum relaxation time $\tau$ in the weak localization experiments with disordered metals is typically $10^{-16}-10^{-14}$ sec, we will have to believe that the phase-breaking time due to electron-electron scattering should be $T$-independent in $2 \mathrm{~d}$ and $3 \mathrm{~d}$ conductors at all temperatures below the melting point! This estimate for $\tau_{\varphi}$ is also much smaller than the electronphonon scattering time $\tau_{e-p h}$ in the temperature range $T<20 K$, which is typical for the experimental studies of the quantum corrections to the conductivity. In this situation, the $T$-dependent weak localization corrections to the resistance of $2 \mathrm{~d}$ and $3 \mathrm{~d}$ conductors should be non-observable. For $1 \mathrm{~d}$ and $2 \mathrm{~d}$ conductors, the strong-localization regime should be non-observable either. Both corollaries of Eqs. (81) of Ref. 9 are in a profound contradiction with the bulk of experimental data, which we review below.

\section{Three-dimensional conductors.}

The study of the WL corrections to the conductivity of $3 \mathrm{~d}$ systems has been focused mostly on thick $\left(a \gg L_{\varphi}\right)$ disordered metal films, metal glasses, and heavily doped semiconductors (for reviews, see Refs. 37 39). For all these 
systems, the well-pronounced temperature dependence of the WL corrections has been observed. From the analysis of the WL magnetoresistance, the temperature dependence of the phase-hreaking time has been extracted. The typical $\tau_{\varphi}(T)$ dependences, obtained for 3d disordered films of $\mathrm{Cu} 44$ and $\mathrm{Cu}-\mathrm{Ge} 41$, are shown in Fig. 11. The electron-phonon scattering governs the phase-relaxation in these $3 d$ conductors over the whole temperature range $T=1.5-40 \mathrm{~K}$. The solid line $\tau_{e-p h} \propto T^{-3}$ corresponds to the electron-phonon relaxation time due to the longitudinal phonons in pure $\mathrm{Cu}$ (see, e.g., Table 3 from Ref. 37). The agreement with the experimental data can be further improved if one takes into account the contribution of transverse phonons, which dominates in disordered metals 21.42 The temperature dependence of $\tau_{\varphi}(3 d)$ due to the electron-electron interactions with the small momentum transfer

$$
\frac{\hbar}{\tau_{e e}}=\frac{\sqrt{2}}{12 \pi^{2} \nu}\left(\frac{k_{B} T}{\hbar D}\right)^{3 / 2}
$$

( $\nu$ is the density of states) is shown in Fig. 11 with the dashed lines. The upper limit of $\tau_{\varphi}$ according to Ref. 9

$$
\frac{\hbar}{\tau_{G Z}^{(3 d)}}=\frac{e^{2}}{3 \pi^{2} \sigma \sqrt{2 D} \tau^{3 / 2}}
$$

( $\sigma$ is the conductivity, and $\tau$ is the elastic scattering time) is shown in Fig. 11 with the dotted lines; this estimate is much smaller than the experimentally measured time. The discrepancy here is as large as 5 (!) orders of magnitude.

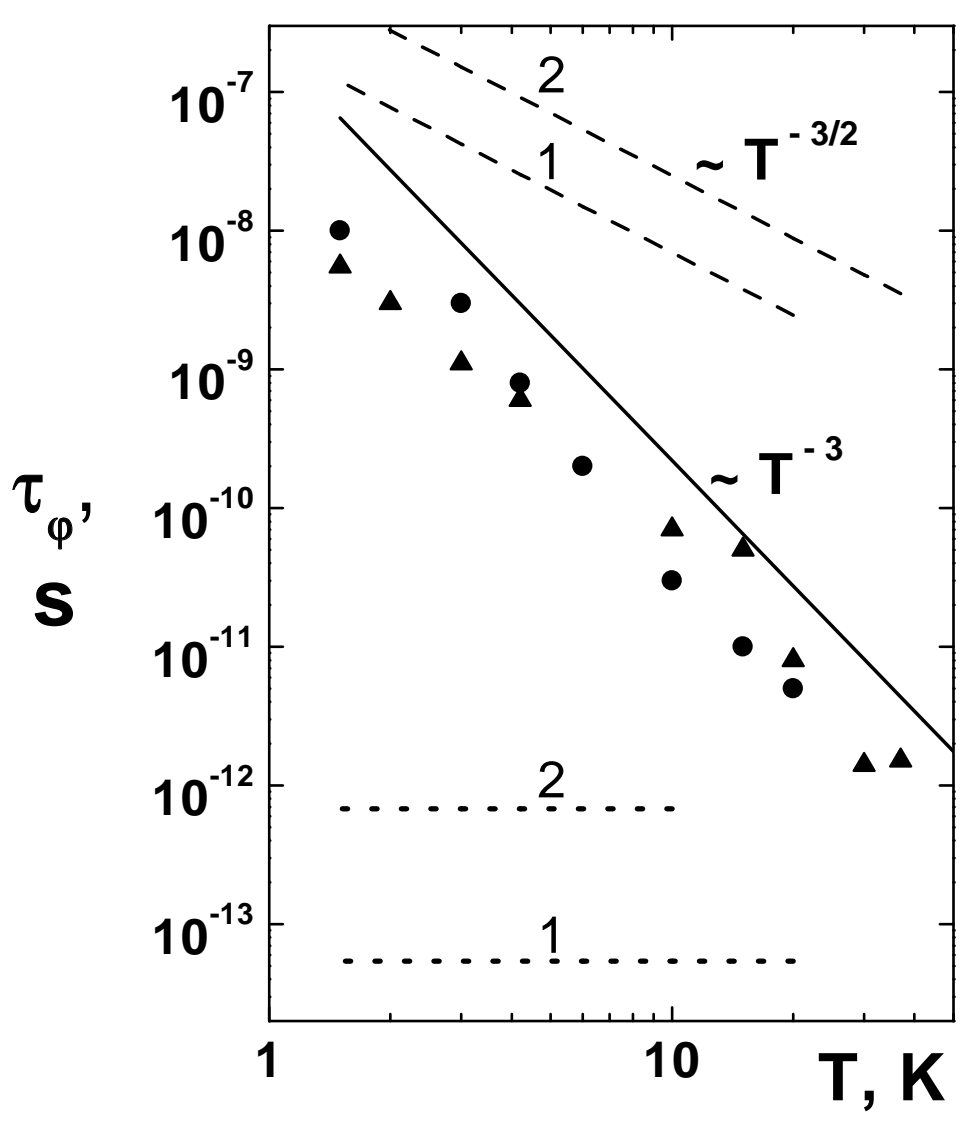


FIG. 11. The temperature dependence of $\tau_{\varphi}$ in $3 d$ metal films: $\bigcirc$ - disordered $\mathrm{Cu}$ films (1) with $\rho=6.6 \cdot 10^{-5} \Omega \cdot \mathrm{cm}$, $D=3.9 \mathrm{~cm}^{2} / \mathrm{s}, \tau=4.8 \cdot 10^{-16} \mathrm{~s}$, Ref. 40; $\triangle-C u_{0.9} G e_{0.1}$ films $(2)$ with $\rho=2.8 \cdot 10^{-5} \Omega \cdot \mathrm{cm}, D=9.3 \mathrm{~cm}^{2} / \mathrm{s}, \tau=1.1 \cdot 10^{-15} \mathrm{~s}$, Ref. 41. The solid line: $\tau_{e-p h}($ pure $\mathrm{Cu})=2.2 \cdot 10^{-7} / T^{3} s$. The dashed lines: $\tau_{e e}(3 d)$, Eq. 6.6) for these samples 1 and 2 respectively. The dotted lines: the maximum $\tau_{\varphi}(3 d)$ according to Eq. (6.7).

Similar discrepancy between the experimental values of $\tau_{\varphi}$ and predictions of Golubev and Zaikin 9 has been observed for metal glasses (see, e.g. Refs. 38,43 45). For example, for $\left(C a_{80} A l_{2 p}\right)_{97.1} A u_{2.9}$ with $\rho=1.3 \cdot 10^{-4} \Omega \cdot \mathrm{cm}$ and $D \simeq 1$ $\mathrm{cm}^{2} / \mathrm{s}$ the experimental value of $\tau_{\varphi}(2.5 K)=7.5 \cdot 10^{-10} s$ was observed 45 . On the other hand, from Eq. (6.7) expect $\tau_{\varphi}<4 \cdot 10^{-14} \mathrm{~s}$. For $Y_{80} S i_{20}$ with $\rho=5 \cdot 10^{-4} \Omega \cdot \mathrm{cm}, \tau \simeq 1 \cdot 10^{-16} \mathrm{~s}$ and $D \simeq 0.7 \mathrm{~cm}^{2} / \mathrm{s}$, Bieri et al. .4 reported $\tau_{\varphi}(4.2 K) \simeq 2 \cdot 10^{-11} s$, whereas Eq. (6.7) gives $\tau_{\varphi} \simeq 3 \cdot 10^{-16} s$.

\section{Two-dimensional conductors.}

The weak localization regime.- Figures 12 and 13 show the typical temperature dependences of $\tau_{\varphi}$ in $2 d$ metal films 44 and GaAs - AlGaAs heterostructures 57 , correspondingly. At low temperatures $(T<1-5 K)$, the phasebreaking is governed by the quasi-elastic electron-electron scattering (the Nyquist phase breaking). The experimental values of $\tau_{\varphi}$ are usually half as large as the estimate of $\tau_{\varphi}(2 d)$, given by Eq. (2.38b).

On the other hand, the experimental data for $\tau_{\varphi}$ exceed by far the upper limit of $\tau_{\varphi}(2 d)$ according to Eq. (81) of Ref. 9

$$
\frac{\hbar}{\tau_{G Z}(2 D)}=\frac{e^{2}}{4 \pi \sigma_{2} \tau}
$$

The discrepancy of Eq. (6.8) with the experiment is especially pronounced for the most disordered samples (4 orders of magnitude for cryo-deposited $\mathrm{Mg}$ films at $\mathrm{T}=0.1 \mathrm{~K}$, Ref. 47). 


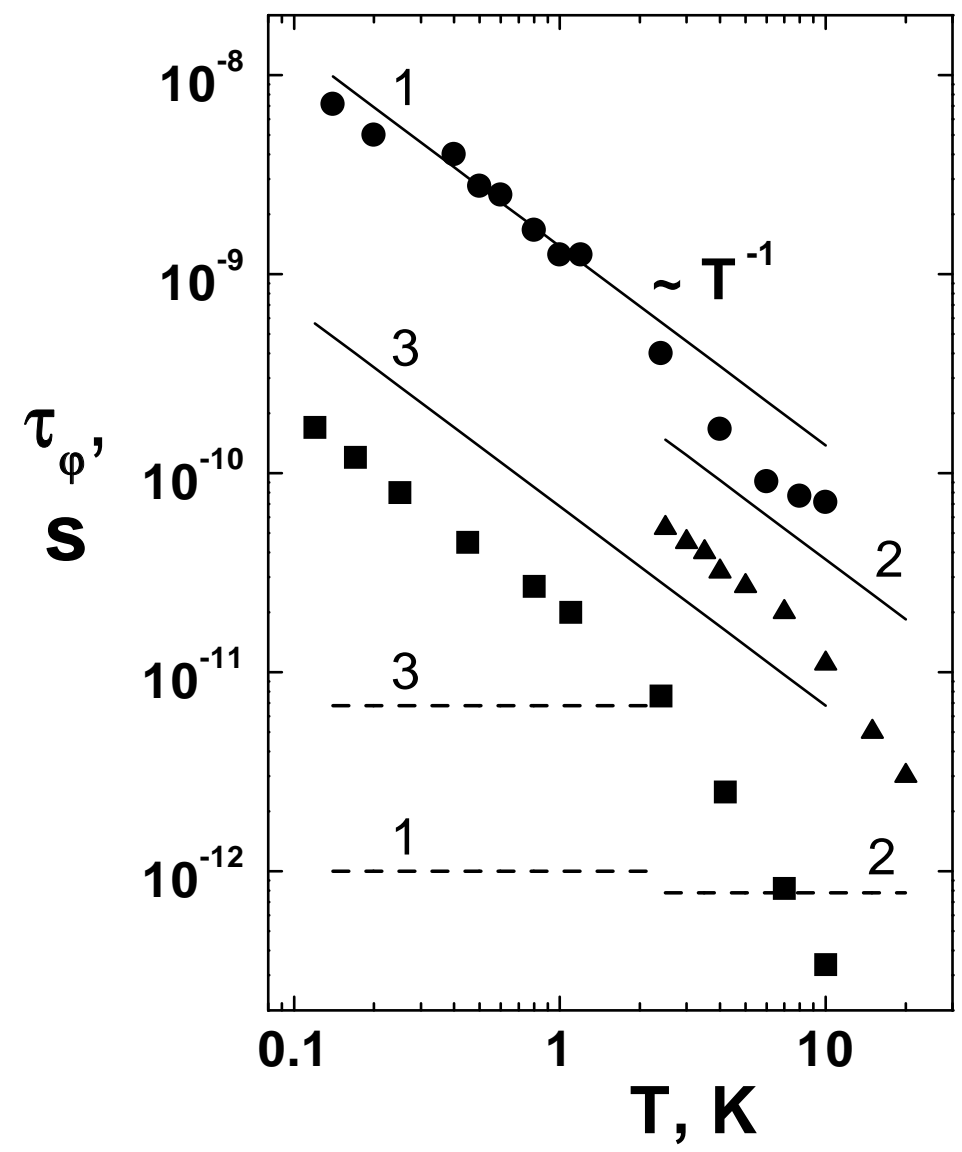

FIG. 12. Themperature dependence of $\tau_{\varphi}$ in $2 d$ metal films: $1(\bigcirc)-M g$ film with $R_{\square}=22.3 \Omega, D=3.8 \mathrm{~cm} / \mathrm{s}$, $\tau=4.6 \cdot 10^{-16} s 472\left(\triangle{ }_{4} A l\right.$ film with $R_{\square}=112 \Omega, D=10.4 \mathrm{~cm}^{2} / \mathrm{s}, \tau=1.7 \cdot 10^{-15} \mathrm{~s} ; 4^{9} 3(\square)-B i$ film, $R_{\square}=630 \Omega, D=8$ $\mathrm{cm}^{2} / \mathrm{s}, \tau=2.8 \cdot 10^{-14} \mathrm{~s} .6$ The solid lines: the temperature dependences of $\tau_{\varphi}(2 d)$ [Eq. (2.38b)] for these samples. The dashed lines: the maximum $\tau_{G Z}(2 d)$ according to Eq. (6.8.

The strong localization regime. With increasing the sheet resistance of $2 d$ samples up to $\simeq 30 k \Omega$, the crossover to the strong localization regime has been observed for both quasi- $2 d$ metal films and $2 d$ electron gas in semiconductor structures (see, f.i., Refs. 50 54). A little extension of the Golubev-Zaikin logic leads one to the conclusion that the "zero point motion" also suppresses the interaction correction to the conductivity since the latter is also caused by the interference, see Sec. IIB. As the result, the conductivity should be temperature-independent at $T \lesssim T_{G Z}=$ $\hbar / \tau_{G Z}(2 d)$. For all the samples studied, $T_{G Z}$ is very large; for example, the samples of Refs. 51,53 are characterized with $T_{G Z} \simeq 10^{5} \mathrm{~K}$. On the other hand, all the crossovers to the strong localization were observed at temperatures of the order of $1 K-10 K$. These experimental data rule out the statement by Golubev and Zaikin that "...strong localization does not take place at all and the $1 d$ and $2 d$ metals do not become insulators even at $T=0 "$. 


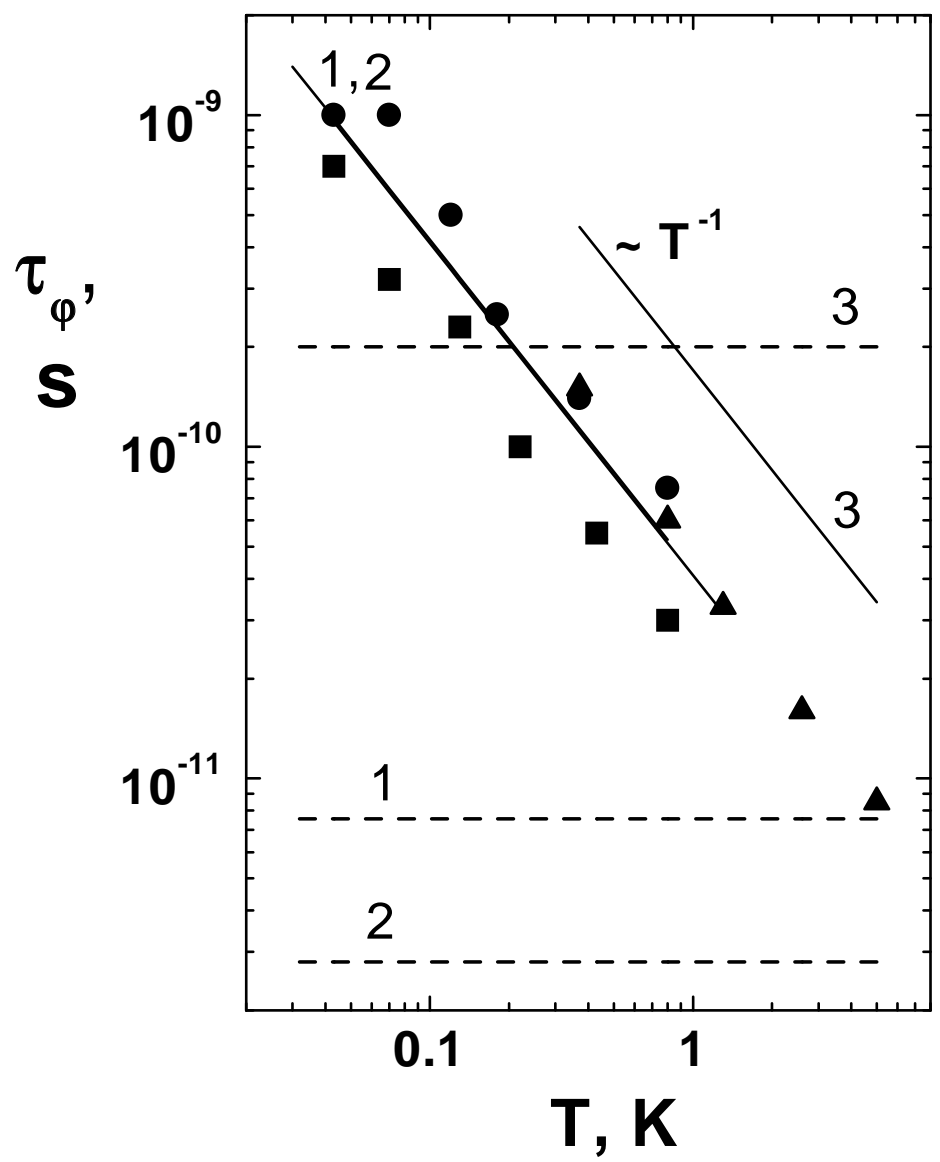

FIG. 13. The temperature dependence of $\tau_{\varphi}$ in $2 \mathrm{~d} G a A s-A l_{x} G a_{1-x} A s$ heterostructures $57: 1(\square)-n=0.87 \cdot 10^{11} \mathrm{~cm}^{-2}$; $R_{\square}=4.3 \mathrm{k} \Omega, D=51 \mathrm{~cm}^{2} / \mathrm{s}, \tau=6.3 \cdot 10^{-13} \mathrm{~s} ; 2(\bigcirc)-n=2.86 \cdot 10^{11} \mathrm{~cm}^{-2} ; R_{\square}=4.0 \mathrm{k} \Omega, D=55 \mathrm{~cm}^{2} / \mathrm{s}, \tau=2.1 \cdot 10^{-13} \mathrm{~s} ; 3$ $(\triangle)-n=7.11 \cdot 10^{11} \mathrm{~cm}^{-2} ; R_{\square}=300 \Omega, D=753 \mathrm{~cm}^{2} / \mathrm{s}, \tau=1.1 \cdot 10^{-12} \mathrm{~s}$. The solid lines: the temperature dependences of $\tau_{N}(2 D)$ [Eq. (2.38b)] for these samples. The dashed lines: the maximum $\tau_{\varphi}(2 d)$ according to Eq. 6.8.

\section{One-dimensional conductors.}

In Ref. 11, Golubev and Zaikin compared their results with the experiment by Khavin et al. 00 and claimed a convincing agreement. We will show below that in fact, the agreement exists only in the temperature region where their result coincides with the conventional one27. Outside this region the comparison becomes meaningless because of the observed crossover to the strong localization regime.

The weak localization regime.- In one dimension, the expression for $\tau_{\varphi}(1 d)$ obtained by Golubev and Zaikin 8 , see Eq. (77) of Ref. 9 or Eq. (10) of Ref. 8, is

$$
\frac{\hbar}{\tau_{G Z}(1 d)}=\frac{e^{2}}{\pi \sigma_{1}} \sqrt{\frac{2 D}{\tau}}\left[1+\frac{2 k_{B} T}{\hbar} \sqrt{\tau_{G Z} \tau}\right] .
$$

In the temperature range $k_{B} T \gg \hbar / \sqrt{\tau_{\varphi} \tau}$, this expression coincides up to a numerical factor of the order of unity with the Nyquist phase-breaking time in $1 \mathrm{~d} 27$ see Eq. (2.38a).

It has been established experimentally that the phase breaking in $1 \mathrm{~d}$ conductors (both metal wires and semiconductor structures) is well described by $\tau_{\varphi}(1 d)$ over a wide temperature range (typically, below several Kelvin) (see, e.g. 
Refs. 55 60). Thus, no wonder that the expression $(6.9)$ is in accord with the experimental data in the regime where the "new theory" coincides with the old-fashioned Eq. (2.38a). We would like to point out again that the explicit comparison of $1 / \tau_{G Z}$ with the experiment is meaningful only if the analytic expression for the magnetoresistance is provided. Since Golubev and Zaikin were not able to obtain such formula, similar to Eq. (2.42d), the procedure of comparison of their results with $\tau_{\varphi}$ extracted with the help of Eq. $(2.42 \mathrm{c})$ is at least problematic.

On the other hand, in the temperature range $k_{B} T \ll \hbar \sqrt{\tau_{\varphi} \tau}$, Golubev and Zaikin predict saturation of the phase breaking length:

$$
\frac{L_{G Z}}{\xi}=\left(\frac{3}{2 \pi^{2}}\right)^{1 / 4} \frac{1}{\sqrt{N}}
$$

where $N$ is the number of the transverse channels in the wire, $L_{G Z}=\sqrt{D \tau_{G Z}}$, and $\xi$ is the $1 \mathrm{~d}$ localization length. Once again, we do not know what the numerical factor in this formula means, since Golubev and Zaikin did not derive the explicit formula for the conductance. [In fact, Eq. (6.9) seems to be erroneous even within the GolubevZaikin logic: the contribution to the zero-temperature term comes from the wave-vectors of the order of the inverse elastic mean free path $1 / l$. Therefore, to be consistent with the "new theory", one "should" use for a wire with the cross-sectional dimensions greater than the elastic mean free path either two- or three- dimensional formulas (6.8) and (6.7), depending on the structure of the wire.]

Anyway, the authors of Ref. 11 use Eq. (6.9) for the analysis of the data. In the regime where deviations from the conventional formula (2.38a ) can be expected from Eq. (6.9), the information on $\tau_{\varphi}$ can not and was not obtained from the experiment, because the samples were already in the strong localization regime.

The strong localization regime.- It has been shown recently that the $1 \mathrm{~d}$ wires fabricated from $\mathrm{Si} \delta$-doped GaAs, demonstrate the crossover from weak to strong localization with decreasing the temperature61.62. This crossover is driven by both localization and interaction effects 60 ; in particular, the importance of the localization effects has been demonstrated by observation of (1) the shift of the crossover by the weak magnetic field; (2) the orbital magnetoresistance on the insulating side of the crossover, and (3) the doubling of the localization length in strong magnetic fields. These experimental facts argue against the conclusion of Golubev and Zaikin "strong localization does not take place at all and the $1 \mathrm{~d}$ and $2 \mathrm{~d}$ metals do not become insulators even at $T=0 "$.

\section{CONCLUSION}

This paper is devoted to the quantum transport of electrons in disordered conductors in the weak localization regime. The motivation to write a paper on the subject that was developed almost 15 years ago was caused by recent experimental and theoretical attempts to reconsider the existing theory of destruction of the one-particle phase coherence (dephasing) in a system of degenerate interacting fermions. It was proposed that, in contrast to basic conclusions of this theory, the dephasing rate remains finite even at zero temperature due to the zero-point oscillations of the electric field.

We criticized this proposal in details from several points of view.

1. We recalled and discussed in details the physical picture of the quantum corrections to the properties of the disordered conductors and tried to demonstrate that such a revision looks absurd from the qualitative point of view.

2. We made a straightforward calculation of the first order in the e-e interaction correction to the conductivity at low dimensions in strong magnetic fields, taking into account all the relevant diagrams. Results of this calculation, see Eqs. (4.13), are in a perfect agreement with the "old" theory and explicitly contradict the zero-point dephasing calculations.

3. We highlighted the sources of the mistakes of the new "theory". The errors were found to be due to the misuse of the semiclassical approximation in disorder averaging.

4. We reviewed existing experiments on magnetoresistance in $3 d, 2 d$, and $1 d$ dimensions, and demonstrated that the measured dephasing rate is sometimes more than five orders of magnitude smaller than the limit, which follows from the zero-point-motion "theory".

We really hope that arguments presented are sufficiently strong to stop the "theoretical" discussion of the zero temperature dephasing.

Our paper also contains the new explicit formulas for the effect of the interaction on the weak localization due to the elastic scattering. Such effects, are shown to be $1 / g^{2}$ corrections which are smaller than the main terms considered 
by the conventional theory everywhere except the vicinity of the metal-insulator crossover. We should warn the practitioners of the fief that in addition to the extra terms in Eq. 4.13) the first order correction to the conductivity in the Cooper channelli and the second order weak localization correction to the conductivity 14 has to be taken into account for comparison with the experiment.

\section{ACKNOWLEDGMENTS}

Useful discussions with M.Yu. Reyzer are gratefully acknowledged. We are thankful to ICTP Trieste for kind hospitality. Part of the work performed in Ruhr-Universität-Bochum was supported by SFB 237 "Unordnung und grosse Fluktuationen". I.A. is A.P. Sloan research fellow.

\section{APPENDIX A: DERIVATION OF IDENTITIES 5.15}

Let us perform the gauge transformation of the Green function

$$
G^{R}\left(\epsilon, \boldsymbol{r}_{1}, \boldsymbol{r}_{2}\right) \rightarrow e^{i e \boldsymbol{A} \cdot\left(\boldsymbol{r}_{1}-\boldsymbol{r}_{2}\right)} G^{R}\left(\epsilon, \boldsymbol{r}_{1}, \boldsymbol{r}_{2}\right)
$$

where $\boldsymbol{A}$ is an arbitrary vector independent of the coordinates. Under this transformation, the exact one-electron Hamiltonian changes according to the rules

$$
\hat{H} \rightarrow \hat{H}-\hat{\boldsymbol{j}} \boldsymbol{A}+\frac{e^{2} \boldsymbol{A}^{2}}{2 m} .
$$

where $\hat{\boldsymbol{j}}$ is the current operator (5.10).

Therefore, it follows from the definition of the Green function that the relation

$$
e^{i e \boldsymbol{A} \cdot\left(\boldsymbol{r}_{1}-\boldsymbol{r}_{2}\right)}\left[\frac{1}{\epsilon-\hat{H}+i 0}\right]_{12}=\left[\frac{1}{\epsilon-\hat{H}+\hat{j} \boldsymbol{A}-\frac{e^{2} \boldsymbol{A}^{2}}{2 m}+i 0}\right]_{12}
$$

holds. Expanding both sides of Eq. (A3) up to the second order in $\boldsymbol{A}$, we obtain Eqs. (5.15).

${ }^{1}$ B.L. Altshuler and A.G. Aronov, in Electron-Electron Interaction in Disordered Systems, edited by A.L. Efros and M. Pollak (North-Holland, Amsterdam, 1985).

2 B.L. Altshuler, A.G. Aronov, M.E. Gershenson, and Yu. V. Sharvin, Sov. Sci, A. Phys, 9, 223 (1987).

${ }^{3}$ P.A. Lee and T.V. Ramakrishnan, Rev. Mod. Phys., 57, 287 (1985).

${ }^{4}$ G. Bergman, Physics Reports, 107, 1 (1984).

${ }^{5}$ B.L. Altshuler, A.G. Aronov, D.E. Khmelnitskii, and A.I. Larkin, in Quantum theory of solids, edited by I.M. Lifshitz, (Mir Publishers, Moscow, 1982).

${ }^{6}$ P. Mohanty, E.M.Q. Jarivala, and R.A. Webb, Phys.Rev.Lett, 78, 3366 (1997).

${ }^{7}$ P.Mohanty and R.A. Webb, Phys. Rev. B55, 13452 (1997).

${ }^{8}$ D.S. Golubev and A.D. Zaikin, Phys. Rev. Lett, 811074 (1998).

${ }^{9}$ D.S. Golubev and A.D. Zaikin, cond-mat/9712203.

${ }^{10}$ B.L. Altshuler, M.E. Gershenson, and I.L. Aleiner, cond-mat/9803125.

${ }^{11}$ D.S. Golubev and A.D. Zaikin, cond-mat/9804156.

${ }^{12}$ R.A. Chentsov, Zh. Exp. Teor. Fiz., 1 8, 374 (1948).

13 B.L. Altshuler and P.A. Lee, Physics Today, 41, 36 (1988).

${ }^{14}$ L.P. Gorkov, A.I. Larkin and D.E. Khmelnitskii, Pis'ma Zh. Eksp. Teor. Fiz. 30, 248 (1979) [JETP Lett. 30, 248 (1979)].

${ }^{15}$ Y. Aharonov and D. Bohm, Phys. Rev. 115, 485 (1959).

${ }^{16}$ B.L. Altshuler, D.E. Khmelnitskii, A.I. Larkin, and P.A. Lee, Phys. Rev. B 22, 5142 (1980).

${ }^{17}$ In order to avoid confusion, we emphasize that the term "one dimensional diffusive system" means that transverse size of the system $a$ is much larger than the Fermi wavelength $\lambda_{F}$ but much smaller than the magnetic length $\lambda_{H}$ and the phase breaking length $L_{\varphi}$. Notion of Luttinger liquid is not applicable here at all. 
18 M.C. Gutzwiller, Chaos in Classical and Quantum Mechanics, (Springer-Verlag, New York, 1990).

${ }^{19}$ D.J. Thouless, Phys. Rev. Lett. 39, 1167 (1977).

${ }^{20}$ A.Schmid, Z. Phys. 271, 251 (1973).

${ }^{21}$ M. Reyzer and A.V. Sergeev, Sov. Phys. JETP, 65, 616 (1986).

22 B.L. Altshuler and A.G. Aronov, JETP Lett., 30, 482 (1979).

${ }^{23}$ B.L. Altshuler, Y. Gefen, A. Kamenev, L.S. Levitov, Phys. Rev. Lett. 78, 2803 (1997).

${ }^{24}$ V. Prigodin and B.L. Altshuler, preprint cond-mat/9703071.

${ }^{25}$ U. Sivan, Y. Imry and A. Aronov, Europhys. Lett. 28, 115 (1994).

${ }^{26}$ E. Abrahams, P.W. Anderson, P.A. Lee, and T.V. Ramakrishnan, Phys. Rev. B24, 6783 (1981).

${ }^{27}$ B.L. Altshuler, A.G. Aronov, and D.E. Khmelnitskii, J. Phys. C 15, 7367 (1982).

28 A. Stern, Y. Aharonov, and Y. Imry, Phys. Rev. A , 41, 3436 (1990).

29 The difference in numerical factor of 2 in Eqs. 2.38a) and (2.42c) in comparison with Eqs. (4.33) and (4.35) of Ref. 1 is caused by an algebraic error in the latter reference. Note, that the experimental data were fitted without taking this into account.

${ }^{30}$ S. Chakravarty and A. Schmid, Phys. Rep. 140, 193 (1986).

${ }^{31}$ In fact, the appearance of $\left(\operatorname{coth} \frac{\omega}{2 T}+\tanh \frac{\epsilon-\omega}{2 T}\right)$ in the expression for $1 / \tau_{\varphi}$ is well known, contrary to the statement of Ref. 9 , see H. Fukuyama and E. Abrahams, Phys. Rev. B 27, 5976 (1983); M.Y. Reyzer, Phys. Rev. B 45, 12949 (1992).

${ }^{32}$ L.V. Keldysh, Zh. Eksp. Teor. Fiz. 47, 1945 (1964) [Sov. Phys. JETP, 20, 1018 (1964)]; we use here the electron Keldysh matrices in notation of A.I. Larkin and Yu.N. Ovchinnikov in Nonequilibrium Superconductivity, edited by D.N. Langenberg and A.I. Larkin, (Elsevier, Amsterdam, 1986).

${ }^{33}$ A.A. Abrikosov, L.P. Gorkov, and I.E. Dzyaloshinskii, Methods of Quantum Field Theory in Statistical Physics, (PrenticeHall, Englewood Cliffs, NJ, 1963).

${ }^{34}$ K.B. Efetov, Supersymmetry in Disorder and Chaos, (Cambridge University Press, New York, 1997).

${ }^{35}$ A.I. Larkin and Yu.N. Ovchinnikov, Zh. Eksp. Teor. Fiz, 55, 2262 (1968) [Sov. Phys. JETP 28, 1200 (1969)].

${ }^{36}$ I.L. Aleiner and A.I. Larkin, Phys. Rev. B, 54, 14423 (1996).

${ }^{37}$ B. L. Altshuler, A. G. Aronov, M. E. Gershenson, and Yu. V. Sharvin, in Sov. Phys. Rev. A9, 223 (1987).

${ }^{38}$ J. S. Dugdale, The electrical properties of disordered metals, Cambridge University Press, 1995.

39 T. A. Polyanskaya and Yu. V. Shmartsev, Sov. Phys. - Semicond. 23, 1 (1989).

${ }^{40}$ A. G. Aronov, M. E. Gershenson, and Yu. E. Zhuravlev, Sov. Phys. - JETP 60, 554 (1984).

${ }^{41}$ W. Eschner, W. Gey, and P. Warnecke, in Proc. of the 17-th Int. Conf. on Low Temp., eds. U. Eckern, A. Schmid, W. Weber and H. Wuhl, p. 497, Elsevier, 1984.

${ }^{42}$ N. G. Ptitsina et al., Phys. Rev. B 56, 10089 (1997).

${ }^{43}$ A. Sahnoune, J. O. Strom-Olsen, and H. E. Fisher, Phys. Rev. B 46, 10035 (1992).

44 J. B. Bieri, A. Fert, G. Creuzet, and A. Schuhl, J. Phys. F 16, 2099 (1986).

${ }^{45}$ F. M. Mayeya and M. A. Howson, J. Phys. Condens. Matter 4, 9355 (1992).

${ }^{46}$ B. J. F. Lin, M. A. Paalanen, A. C. Gossard, and D. C. Tsui, Phys. Rev. B 29, 927 (1984).

47 A. E. White, R. C. Dynes, and J. P. Garno, Phys. Rev. B 29, 3694 (1984).

${ }^{48}$ F. Komori, S. Kobayashi, and W. Sasaki, J. Phys. Soc. Jpn. 52, 4306 (1983).

${ }^{49}$ M. E. Gershenson, V. N. Gubankov, and Yu. E. Zhuravlev, Sov. Phys. - JETP 58, 167 (1983).

${ }^{50}$ D. J. Bishop, D. C. Tsui, and R. C. Dynes, Phys. Rev. Lett. 44, 1153 (1980).

${ }^{51}$ Z. Ovadyahu and Y. Imry, J. Phys. C 16, L471 (1983).

${ }^{52}$ Y. Liu, B. Nease, K. A. McGreer, and A. M. Goldman, Europhys. Lett. 19, 409 (1992).

53 S.-Y. Hsu and J. M. Valles, Phys. Rev. Lett. 74, 2331 (1995).

${ }^{54}$ F. W. Van Keuls, H. Mathur, H. W. Jiang, and A. J. Dahm, Phys. Rev. B 56, 13263 (1997).

55 T. J. Thornton, M. Pepper, H. Ahmed, D. Andrews, and G. J. Davies, Phys. Rev. Lett. 56, 1198 (1986).

${ }^{56}$ S. Wind, M. J. Rooks, V. Chandrasekhar, and D.E. Prober, Phys. Rev. Lett. 57, 633 (1986).

57 J. J. Lin and N. Giordano, Phys. Rev. B 33, 1519 (1986).

${ }^{58}$ D. M. Pooke, N. Paquin, M. Pepper, and A. Gundlach. J. Phys. Condens. Matter 1, 3289 (1989).

${ }^{59}$ P. M. Echternach, M. E. Gershenson, H. M. Bozler, A. L. Bogdanov, and B. Nilsson. Phys.Rev. B 48, 11516 (1993).

${ }^{60}$ Yu. B. Khavin, M. E. Gershenson, and A. L. Bogdanov, Phys. Rev. Lett. 81, 1066 (1998).

${ }^{61}$ M. E. Gershenson, Yu. B. Khavin, A. G. Mikhalchuk, H. M. Bozler, and A. L. Bogdanov, Phys. Rev. Lett.79, 725 (1997).

${ }^{62}$ Yu. B. Khavin, M. E. Gershenson, and A. L. Bogdanov, Phys. Rev. B (1998). 